\title{
Access to Indole-Annulated Medium-Sized Lactams through Protonation/Deuteration-Induced Ring-Opening of Spiroindolines
}

\author{
Jianhui Qiao, Huili Liu,* and Shaozhong Wang*
}

Cite This: ACS Omega 2021, 6, 25049-25061

Read Online

ABSTRACT: A protocol has been developed to access indoleannulated eight- and nine-membered lactams through protonationinduced ring-opening of spiroindolines, which are dearomative Heck products of tetrahydro- $\beta$-carbolines or hexahydroazepino[3,4- $b]$ indoles. Brønsted acids and nucleophiles were explored and compared in the transformation. A combination of deuterated hydrochloride and deuterated methanol enables deuterative ring-opening of spiroindolines to afford medium-sized lactam diastereoisomers with a deuterium content ratio around 1:1.

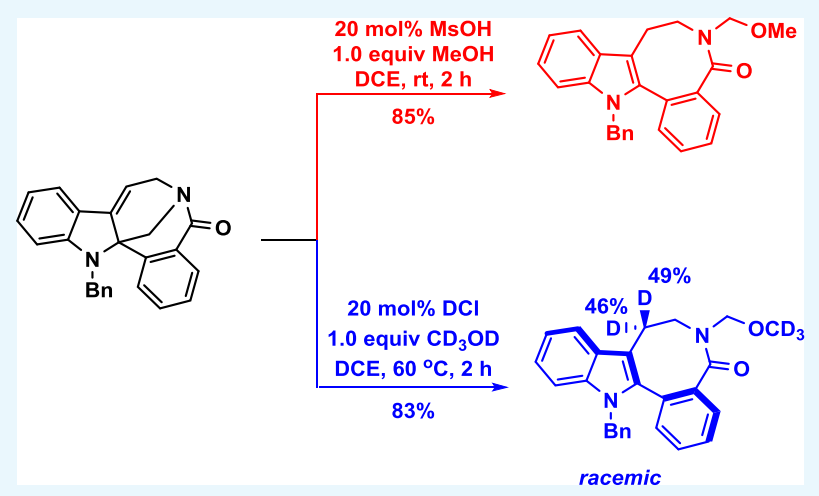

\section{INTRODUCTION}

Indole-annulated medium-sized nitrogen-containing heterocyclic rings are core structures embedded in bioactive natural alkaloids, including balasubramide, ${ }^{1}$ deoxyisoaustamide, ${ }^{2}$ okaramine, ${ }^{3}$ lundurine $\mathrm{A}-\mathrm{D},{ }^{4}$ and vinblastine. ${ }^{5}$ Generally, the construction of medium-sized compounds has been challenging in organic synthesis due to unfavorable entropy and transannular interactions. ${ }^{6}$ It is impressive that significant strategies including cycloaddition, ${ }^{7}$ cycloisomerization, ${ }^{8}$ and ring expansion ${ }^{9}$ have been invented for the generation of eightand nine-membered nitrogen-containing heterocycles.

Recently, we have developed a diastereoselective protocol to prepare indole-annulated eight-membered lactams through light-driven alkylative ring-opening of spiroindolines, a type of dearomative Heck product of easily available $\beta$-carbolines. ${ }^{10}$ DFT calculations and mechanistic experiments support that a cationic $\mathrm{C}-\mathrm{C}$ fragmentation should be favorable rather than a homolytic $\mathrm{C}-\mathrm{C}$ fragmentation, in which a tertiary cation intermediate might be involved (Scheme 1a). ${ }^{11}$ Because a tertiary carbon cation could be formed regioselectively through direct protonation of an alkene, we envisioned an alternative to indole-annulated medium-sized lactams by protonationinduced ring-opening of the spiroindolines (Scheme 1b). The key issues of the transformation include the choice of Brønsted acids, the tolerance of the indole ring, and the nucleophile to trap the iminium intermediate. Furthermore, if this can be realized, a deuterative ring-opening would be feasible by employing deuterated acids and deuterated nucleophiles, which provides an unprecedented protocol to prepare deuterated indole-containing medium-sized hetero-
Scheme 1. Reaction Design

(a) Diastereoselective alkylative ring-opening of spiroindolines

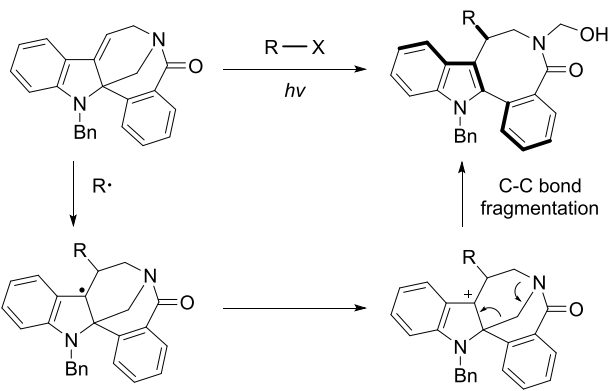

(b) Protonation/Deutration-induced ring-opening of spiroindolines (this work)

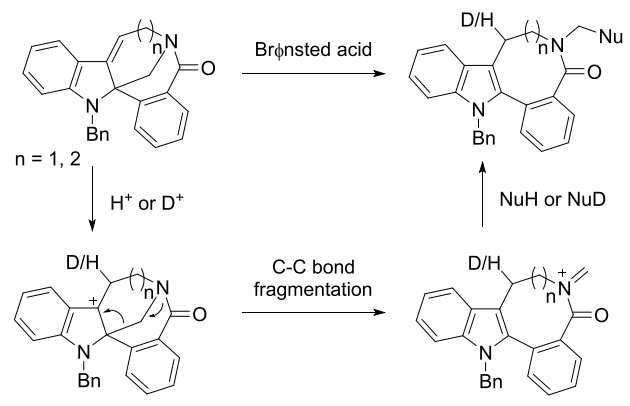

Received: August 9, 2021

Published: September 14, 2021 
cycles. $^{12}$ Here, we report our experimental results on the reaction design.

\section{RESULTS AND DISCUSSION}

We commenced the study by reacting spiroindole 1a with methanol using acetic acid as a promoter. The anticipated ringopening reaction did not take place even after heating in 1,2dichloroethane at $60^{\circ} \mathrm{C}$ for $12 \mathrm{~h}$, probably due to the relatively weak acidity (Table 1 , entry 1 ). However, in the presence of

Table 1. Optimization of the Protonation-Induced RingOpening of Spiroindoline $1 \mathrm{a}^{a}$

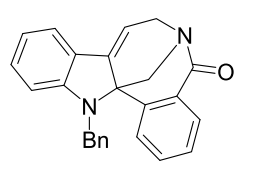

$1 a$
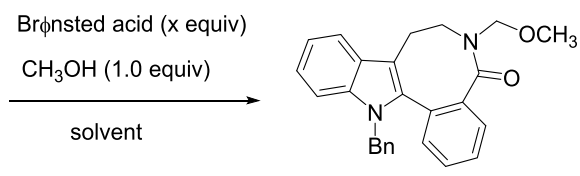

2a

$\begin{array}{clclccc}\text { entry } & \text { acid } & x & \text { solvent } & \text { temp. }\left({ }^{\circ} \mathrm{C}\right) & \text { time }(\mathrm{h}) & \text { yield }(\%)^{b} \\ 1 & \mathrm{HOAc} & 1.0 & \mathrm{DCE} & 60 & 12 & \\ 2 & \mathrm{TFA} & 1.0 & \mathrm{DCE} & 60 & 2 & 68 \\ 3^{c} & \mathrm{HCl} & 1.0 & \mathrm{DCE} & 60 & 2 & 80 \\ 4 & \mathrm{MsOH} & 1.0 & \mathrm{DCE} & \mathrm{rt} & 2 & 89 \\ 5 & \mathrm{MsOH} & 0.2 & \mathrm{DCE} & \mathrm{rt} & 2 & 85 \\ 6 & \mathrm{MsOH} & 0.1 & \mathrm{DCE} & \mathrm{rt} & 12 & 70 \\ 7 & \mathrm{MsOH} & 0.2 & \mathrm{DCM} & \mathrm{rt} & 2 & 81 \\ 8 & \mathrm{MsOH} & 0.2 & \mathrm{CHCl} & \mathrm{rt} & 2 & 63 \\ 9 & \mathrm{MsOH} & 0.2 & \text { dioxane } & \mathrm{rt} & 2 & 25 \\ 10 & \mathrm{MsOH} & 0.2 & \mathrm{THF} & \mathrm{rt} & 2 & 24 \\ 11 & \mathrm{MsOH} & 0.2 & \text { toluene } & \mathrm{rt} & 2 & 58 \\ 12 & \mathrm{MsOH} & 0.2 & \mathrm{MeCN} & \mathrm{rt} & 2 & \\ 13 & \mathrm{MsOH} & 0.2 & \text { DMF } & \mathrm{rt} & 2 & \\ 14 & \mathrm{MsOH} & 0.2 & \text { DMSO } & \mathrm{rt} & 2 & \end{array}$

${ }^{a}$ Reaction conditions: spiroindoline 1 a $(0.05 \mathrm{mmol})$, acid ( $x$ equiv), methanol $(0.05 \mathrm{mmol})$, and degassed solvent $(0.25 \mathrm{~mL})$ were stirred in a sealed vial. ${ }^{b}$ Isolated yield after column purification. ${ }^{c} 20 \% \mathrm{HCl}$ in water.

stronger Brønsted acids such as trifluoroacetic acid, hydrochloride, and methanesulfonic acid, the spiroindoline was converted successfully to eight-membered lactam $\mathbf{2 a}$ (Table 1, entries 2-4). The structure of $2 \mathrm{a}$ was confirmed unequivocally by single-crystal X-ray diffraction (Figure 1). It was impressive that $2 \mathrm{a}$ was isolated in $89 \%$ yield under the promotion of methanesulfonic acid even when the ring-opening reaction was performed at room temperature. Control experiments showed that 0.2 equiv of methanesulfonic acid is appropriate for the transformation (entries 5 and 6). Further screenings of solvents by fixing methanesulfonic acid and methanol demonstrated that lower yields were obtained in dichloromethane, chloroform, 1,4-dioxane, tetrahydrofuran, and toluene, while the reaction was completely inhibited in acetonitrile, $\mathrm{N}, \mathrm{N}$-dimethylformamide, and dimethyl sulfoxide (Table 1, entries 9-14).

The scope of the protonation-induced $\mathrm{C}-\mathrm{C}$ bond fragmentation was next examined (Scheme 2). Like spiroindoline 1a, spiroindolines with electron-donating and electronwithdrawing substituents $(\mathrm{Me}, \mathrm{MeO}, \mathrm{F}, \mathrm{Cl})$ located at ortho, meta, and para positions of the benzamide ring, participated in the ring-opening reaction with methanol smoothly, affording indole-annulated eight-membered lactams $\mathbf{2} \mathbf{b}-\mathbf{2} \mathbf{k}$ in yields ranging from 76 to $90 \%$. It seems that the electron-donating

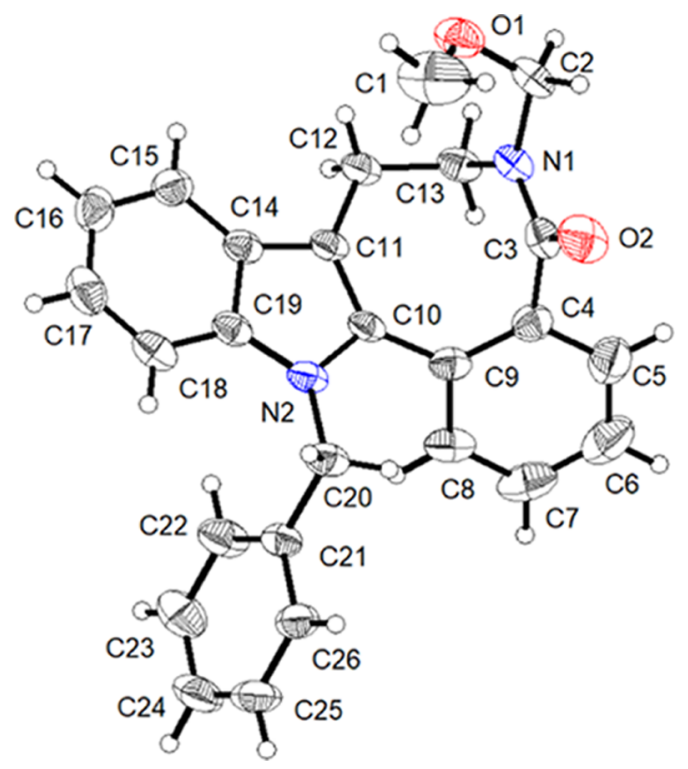

Figure 1. X-ray crystal structure of $\mathbf{2 a}$ (30\% probability levels).

group might be more favorable than the electron-withdrawing group for the transformation. Different substituents $(\mathrm{MeO}, \mathrm{Cl}$, $\mathrm{Me}$ ) on the indole ring were also compatible, affording lactams $\mathbf{2 l - 2 n}$ in a comparable efficiency. Replacing the benzyl group on the nitrogen of the indole ring by a methyl group did not interfere with the conversion, affording lactam 20 in $91 \%$ yield. Spiroindoline 1a reacted with heteroatom-containing nucleophiles with increasing steric hindrance such as ethanol, benzyl alcohol, isopropanol, and 4-methylbenzenethiol in a similar manner, affording lactams $2 \mathrm{p}-2 \mathrm{~s}$ in good yields. Carbon nucleophiles including 2-naphthol and indole engaged in the ring-opening reaction to afford lactams $2 t$ and $2 \mathbf{u}$ in 82 and $70 \%$ yields, respectively.

We further tested the feasibility of deuteration-induced ringopening of spiroindolines by employing commercially available deuterated Brønsted acid and deuterated nucleophile. To our delight, the ring-opening of spiroindoline 1a was observed at $60{ }^{\circ} \mathrm{C}$ under the promotion of deuterated trifluoroacetic acid and deuterated methanol, affording indole-annulated eightmembered lactam $3 a$ in $70 \%$ yield (Table 2 , entry 1 ). The assignment of different hydrogens of lactam 3a was identified by a series of NMR studies. Further NOE experiments of lactam 3a supported that hydrogens $a, d$, and $e$ are close in space, in which the cross-peaks of hydrogen $a$ and $e, a$ and $d$ had strong intensities (Figure 2). Based on the integration of peaks on the ${ }^{1} \mathrm{H}$ NMR spectra of $3 \mathrm{a}$, we found that hydrogens $c$ and $d$ were partially deuterated with 49 and $48 \%$ deuterium installation. When deuterated hydrochloride was employed as Brønsted acid, the medium-sized lactam was isolated in $83 \%$ yield of 49 and $46 \%$ deuterium contents (Table 2, entry 2). It is worth mentioning that the competitive nucleophilic addition of $\mathrm{D}_{2} \mathrm{O}$ toward the iminium intermediate did not happen, highlighting that deuterated methanol performed as a benign nucleophile. Inferior results were obtained in dichloromethane, chloroform, toluene, 1,4-dioxane, and THF, and no conversions were observed in acetonitrile, DMF, and DMSO (Table 1, entries 3-10), which are consistent with those encountered in the protonation-induced ring-opening reaction.

By fixing the combination of deuterated hydrochloride and deuterated methanol, the deuteration-induced ring-opening of 
Scheme 2. Scope of Indole-Annulated Eight-Membered Lactams ${ }^{a}$

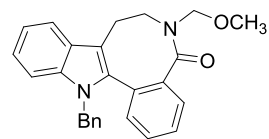

2a, $85 \%$
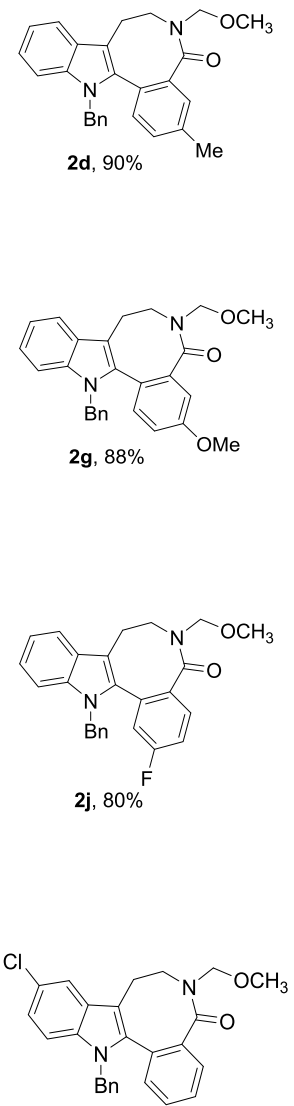

$2 \mathrm{~m}, 73 \%$

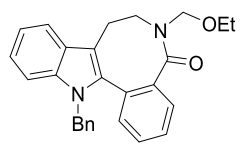

$2 p, 74 \%$

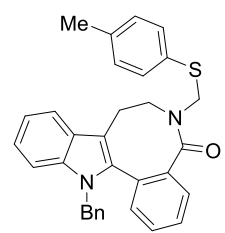

2s, $87 \%$

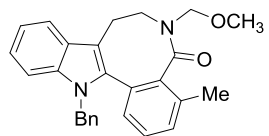

2b, $87 \%$
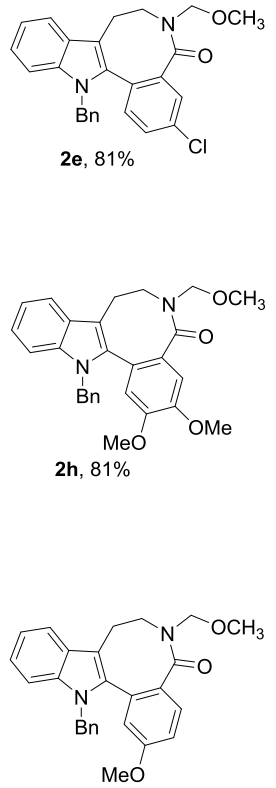

2k. $86 \%$

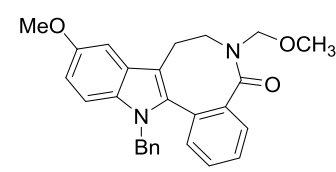

2n, $83 \%$

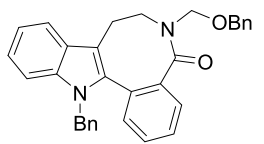

2q, $83 \%$

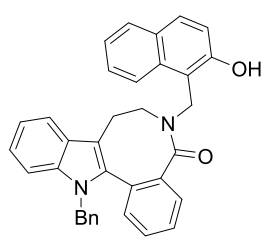

2t, $82 \%$

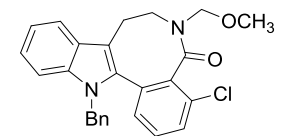

2c, $76 \%$
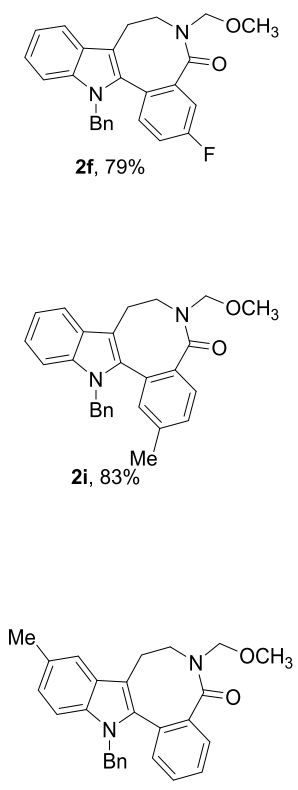

2I, $86 \%$

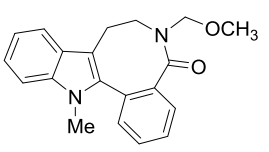

2o, $91 \%$

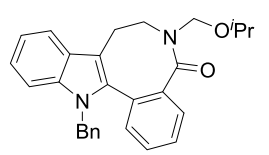

2r, $77 \%$

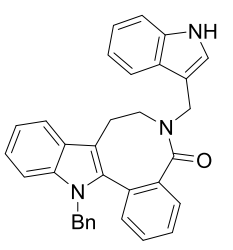

2u, $70 \%$

${ }^{a}$ Reaction conditions: a mixture containing spiroindoline $1(0.05 \mathrm{mmol})$, methanesulfonic acid $(0.01 \mathrm{mmol})$, nucleophile (0.05 mmol), and degassed 1,2-dichloroethane $(0.25 \mathrm{~mL})$ was stirred in a sealed vial at room temperature for $2 \mathrm{~h}$. Isolated yield after column purification.

spiroindoline 1a was extended (Scheme 3). Other candidates with methoxy, methyl, chlorine, and fluorine groups located at different positions on the benzamide and indoline rings as well as the nitrogen of the indoline afforded lactams $\mathbf{3 b} \mathbf{b} \mathbf{3 0}$ in good to excellent yields. The deuterium content ratio of two hydrogens on the eight-membered ring is close to $1: 1$, except for that of compounds $\mathbf{3 b}$ and $\mathbf{3 c}$. It was suggested that in most cases, nondiastereoselective deuteration of the alkene in 
Table 2. Optimization of the Deuteration-Induced Ring-Opening of Spiroindoline $1 a^{a}$

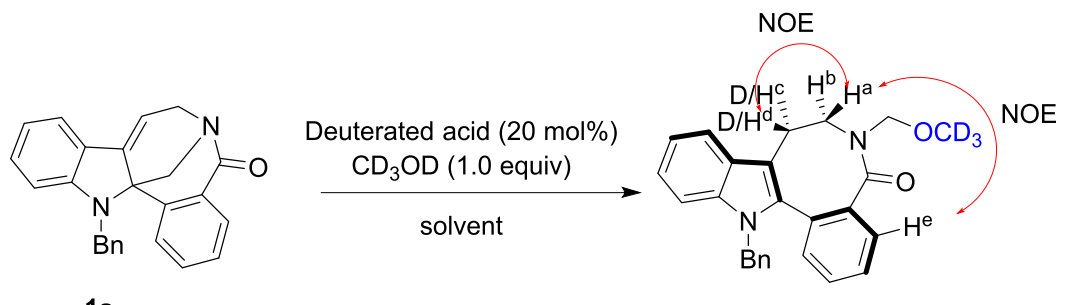

$1 \mathrm{a}$

$\pm 3 a$

\begin{tabular}{|c|c|c|c|c|c|c|c|}
\hline \multirow[b]{2}{*}{ entry } & \multirow[b]{2}{*}{ deuterated acid } & \multirow[b]{2}{*}{ solvent } & \multirow[b]{2}{*}{ temp. $\left({ }^{\circ} \mathrm{C}\right)$} & \multirow[b]{2}{*}{$t(\mathrm{~h})$} & \multirow[b]{2}{*}{ yield $(\%)^{b}$} & \multicolumn{2}{|c|}{ deuterium content } \\
\hline & & & & & & $\overline{H^{c}(\%)^{c}}$ & $\mathrm{H}^{\mathrm{d}}(\%)^{c}$ \\
\hline 1 & $\mathrm{CF}_{3} \mathrm{COOD}$ & DCE & 60 & 2 & 70 & 49 & 48 \\
\hline 2 & $\mathrm{DCl}\left(20 \%\right.$ in $\left.\mathrm{D}_{2} \mathrm{O}\right)$ & DCE & 60 & 2 & 83 & 49 & 46 \\
\hline 3 & $\mathrm{DCl}\left(20 \%\right.$ in $\left.\mathrm{D}_{2} \mathrm{O}\right)$ & $\mathrm{DCM}$ & 40 & 8 & 72 & 48 & 45 \\
\hline 4 & $\mathrm{DCl}\left(20 \%\right.$ in $\left.\mathrm{D}_{2} \mathrm{O}\right)$ & $\mathrm{CHCl}_{3}$ & 60 & 2 & 61 & 45 & 41 \\
\hline 5 & $\mathrm{DCl}\left(20 \%\right.$ in $\left.\mathrm{D}_{2} \mathrm{O}\right)$ & toluene & 60 & 2 & 50 & 45 & 40 \\
\hline 6 & $\mathrm{DCl}\left(20 \%\right.$ in $\left.\mathrm{D}_{2} \mathrm{O}\right)$ & dioxane & 60 & 2 & 28 & 43 & 41 \\
\hline 7 & $\mathrm{DCl}\left(20 \%\right.$ in $\left.\mathrm{D}_{2} \mathrm{O}\right)$ & $\mathrm{THF}$ & 60 & 2 & 29 & 42 & 39 \\
\hline 8 & $\mathrm{DCl}\left(20 \%\right.$ in $\left.\mathrm{D}_{2} \mathrm{O}\right)$ & $\mathrm{MeCN}$ & 60 & 2 & & & \\
\hline 9 & $\mathrm{DCl}\left(20 \%\right.$ in $\left.\mathrm{D}_{2} \mathrm{O}\right)$ & DMF & 60 & 2 & & & \\
\hline 10 & $\mathrm{DCl}\left(20 \%\right.$ in $\left.\mathrm{D}_{2} \mathrm{O}\right)$ & DMSO & 60 & 2 & & & \\
\hline
\end{tabular}

${ }^{a}$ Reaction conditions: spiroindoline $1 \mathrm{a}(0.05 \mathrm{mmol})$, deuterated acid $(0.01 \mathrm{mmol}), \mathrm{CD}_{3} \mathrm{OD}(0.05 \mathrm{mmol}), 1,2$-dichloroethane (degassed, $\left.0.25 \mathrm{~mL}\right)$, $60{ }^{\circ} \mathrm{C}, 2 \mathrm{~h}$. ${ }^{b}$ Isolated yields after column purification. ${ }^{c}$ The deuterium content was calculated by the integration of peaks shown on the ${ }^{1} \mathrm{H}$ NMR spectra.
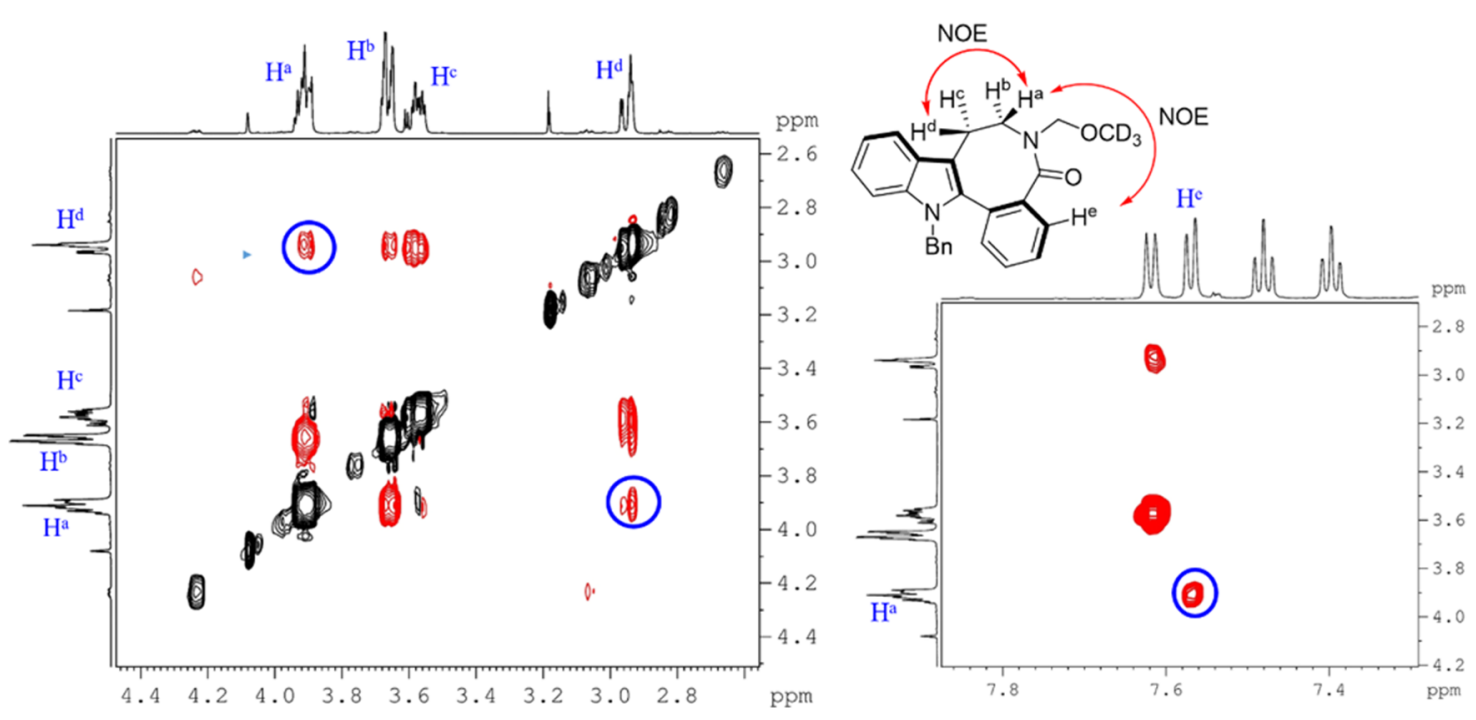

Figure 2. ${ }^{2} \mathrm{D}$ NOESY spectra of compound 3a with a mixing time of $700 \mathrm{~ms}$.

spiroindolines is predominant, while the ortho substituent on the benzamide ring leads to a diastereoselective deuteration, which makes one diastereomer of $\mathbf{3 b}$ or $\mathbf{3} \mathbf{c}$ excess.

Based on the protonation/deuteration-promoted ring-opening reaction, indole-annulated nine-membered lactams $\mathbf{5 a - 5 d}$ can be prepared from spiroindoline 4 (Scheme 4). As illustrated, under the promotion of methanesulfonic acid, spiroindoline 4 reacted with oxygen and carbon nucleophiles to afford lactams $\mathbf{5 a}-\mathbf{5 c}$ in excellent yields, in which methoxy, 2-naphthol, and indole were installed to the side chain of the nine-membered lactams. In the presence of deuterated hydrochloride and methanol, lactam $\mathbf{5 d}$ was obtained in $88 \%$ yield with 58 and $41 \%$ deuterium installation.

\section{CONCLUSIONS}

In summary, we have established a protocol to prepare indoleannulated eight- and nine-membered lactams by taking advantage of protonation-induced ring-opening of spiroindolines. Under the promotion of methanesulfonic acid, spiroindolines reacted with different hetero and carbon nucleophiles to afford medium-sized lactams with side chains containing alkoxy, thiol ether, 2-naphthol, and indole groups. In the presence of deuterated hydrochloride and deuterated methanol, a deuterative ring-opening of spiroindolines proceeded smoothly to afford deuterated medium-sized lactams. Further investigations on the diastereoselective deuteration-induced ring-opening of spiroindolines are underway. 
Scheme 3. Scope of Deuterated Indole-Annulated Eight-Membered Lactams ${ }^{a}$

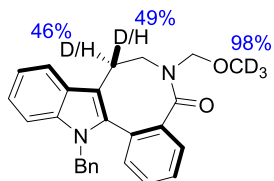

3a, $83 \%$

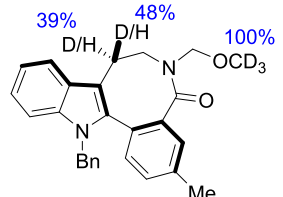

3d, $90 \%$

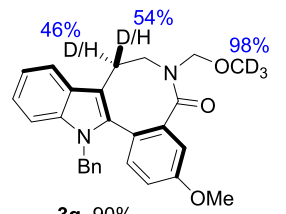

$3 \mathrm{~g}, 90 \%$
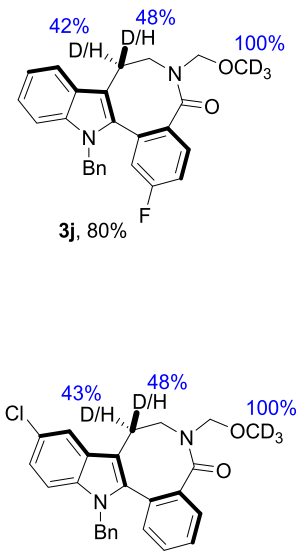

$3 \mathrm{~m}, 69 \%$

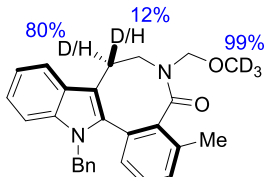

3b, $82 \%$

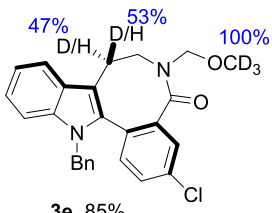

3e, $85 \%$
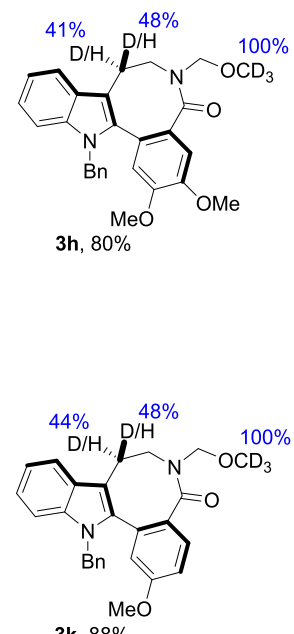

$3 \mathbf{k}, 88 \%$

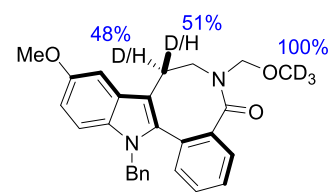

$3 n, 84 \%$

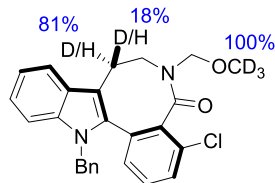

$3 c, 72 \%$

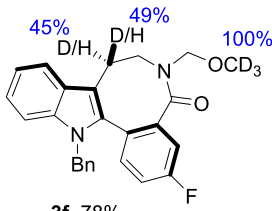

3f, $78 \%$
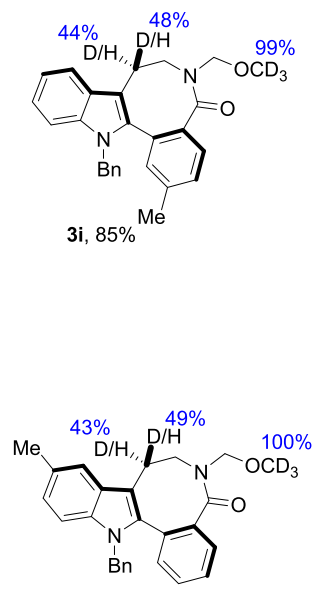

3I, $89 \%$

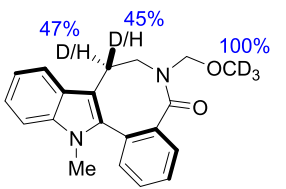

3o, $91 \%$

${ }^{a}$ Reaction conditions: a mixture containing spiroindoline $1(0.05 \mathrm{mmol})$, deuterated hydrochloride $\left(20 \%\right.$ in $\left.\mathrm{D}_{2} \mathrm{O}, 0.01 \mathrm{mmol}\right)$, deuterated methanol $(0.05 \mathrm{mmol})$, degassed 1,2-dichloroethane $(0.25 \mathrm{~mL})$ was stirred at $60{ }^{\circ} \mathrm{C}$ for $2 \mathrm{~h}$. Isolated yield after column purification.

\section{EXPERIMENTAL SECTION}

General Information. ${ }^{1} \mathrm{H}$ NMR spectra was obtained at $400 \mathrm{MHz} .{ }^{2} \mathrm{H} \mathrm{NMR}$ spectra were recorded at a frequency of 92 $\mathrm{MHz} .{ }^{13} \mathrm{C}$ NMR spectra were obtained at $100 \mathrm{MHz}$, and ${ }^{19} \mathrm{~F}$ NMR spectra were obtained at $376 \mathrm{MHz}$. Spectra were recorded in a $\mathrm{CDCl}_{3}$ solution using the residual protonated solvent as the internal standard, and $J$ values are given in hertz. IR spectra were recorded on a Fourier transform infrared spectrometer and listed in $\mathrm{cm}^{-1}$. High-resolution mass spectral analyses (HRMS) were performed on a Q-TOF-MS spectrometer. All air moisture-sensitive reactions were conducted in oven-dried glassware under a nitrogen atmosphere using dry and degassed solvents. Flash column chromatography was performed over silica gel (300-400 mesh). All commercially available reagents were used without further purification. Spiroindolines were prepared according to the known procedure. ${ }^{10}$

General Procedure A for the Synthesis of IndoleAnnulated Medium-Sized Lactams. To a dry $1.5 \mathrm{~mL}$ glass bottle, spiroindoline 1 or $4(0.05 \mathrm{mmol})$ and degassed DCE $(0.25 \mathrm{~mL})$ were added successively. Then, a nucleophile (50 $\mu \mathrm{L}, 0.05 \mathrm{mmol}, 1 \mathrm{~mol} / \mathrm{L}$ DCE solution) and $\mathrm{MsOH}(10 \mu \mathrm{L}$, $0.01 \mathrm{mmol}, 1 \mathrm{~mol} / \mathrm{L}$ DCE solution) were added. The reaction mixture was stirred at room temperature for $2 \mathrm{~h}$. The solution was concentrated under vacuum to afford a residue, which was purified by column chromatography on silica gel to afford lactam 2 or $\mathbf{5 a}-\mathbf{5 c}$. 
Scheme 4. Access to Indole-Annulated Nine-Membered Lactams from Spiroindoline 4

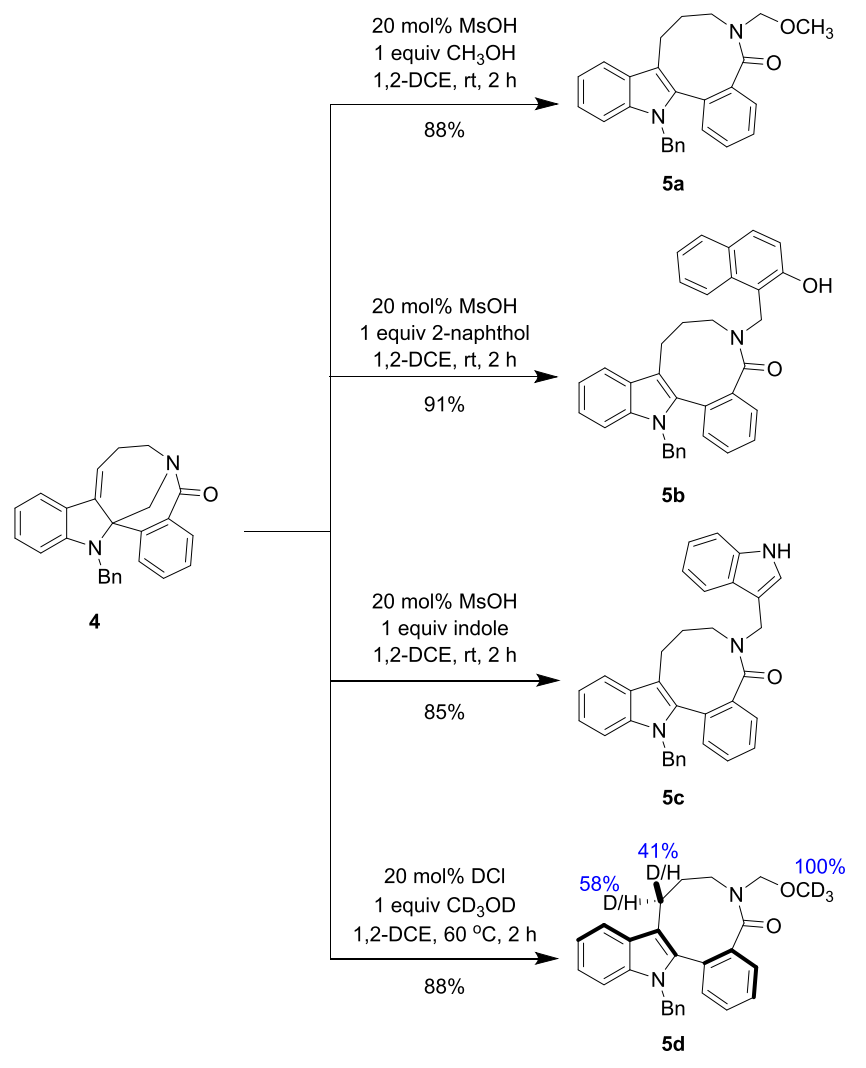

General Procedure B for the Synthesis of Deuterated Indole-Annulated Medium-Sized Lactams. To a dry 1.5 $\mathrm{mL}$ glass bottle, spiroindoline 1 or $4(0.05 \mathrm{mmol})$ and degassed DCE $(0.25 \mathrm{~mL})$ were added successively. Then, $\mathrm{CD}_{3} \mathrm{OD}(50 \mu \mathrm{L}, 0.05 \mathrm{mmol}, 1 \mathrm{~mol} / \mathrm{L} \mathrm{DCE}$ solution) and $\mathrm{DCl}$ ( $20 \%$ in $\mathrm{D}_{2} \mathrm{O}, 2 \mu \mathrm{L}, 0.01 \mathrm{mmol}$ ) were added. The reaction mixture was stirred at $60{ }^{\circ} \mathrm{C}$ for $2 \mathrm{~h}$. The solution was concentrated under vacuum to afford a residue, which was purified by chromatography on silica gel to afford lactam 3 or $5 d$.

13-Benzyl-6-(methoxymethyl)-6,7,8,13-tetrahydro-5Hbenzo[6,7]azocino[5,4-b]indol-5-one (2a). According to procedure A, lactam 2a was obtained from spiroindoline 1a (18.2 $\mathrm{mg}, 0.05 \mathrm{mmol}$ ) and methanol (1.65 mg, $0.05 \mathrm{mmol})$ as a pale yellow solid $(16.3 \mathrm{mg}, 85 \%$ yield, eluent: petroleum ether/ethyl acetate $=3 / 1)$. Mp 101-103 ${ }^{\circ} \mathrm{C} .{ }^{1} \mathrm{H}$ NMR (400 $\left.\mathrm{MHz}, \mathrm{CDCl}_{3}\right) \delta 7.61-7.50(\mathrm{~m}, 2 \mathrm{H}), 7.44(\mathrm{td}, J=7.5,1.3 \mathrm{~Hz}$, $1 \mathrm{H}), 7.36(\mathrm{td}, J=7.6,1.5 \mathrm{~Hz}, 1 \mathrm{H}), 7.28-7.11(\mathrm{~m}, 7 \mathrm{H}), 6.99-$ $6.94(\mathrm{~m}, 2 \mathrm{H}), 5.17(\mathrm{~d}, J=16.9 \mathrm{~Hz}, 1 \mathrm{H}), 5.10-4.98(\mathrm{~m}, 2 \mathrm{H})$, $4.88(\mathrm{~d}, J=10.3 \mathrm{~Hz}, 1 \mathrm{H}), 3.88(\mathrm{td}, J=14.2,4.6 \mathrm{~Hz}, 1 \mathrm{H})$, 3.68-3.49 (m, 2H), 3.15 (s, 3H), 2.92 (ddd, $J=16.5,4.4,1.2$ $\mathrm{Hz}, 1 \mathrm{H}) \mathrm{ppm} .{ }^{13} \mathrm{C} \mathrm{NMR}\left(100 \mathrm{MHz}, \mathrm{CDCl}_{3}\right) \delta 172.3,138.0$, $137.6,137.5,134.0,130.6,129.3,129.2,129.0,128.7,128.6$, 128.2 , 127.2, 126.1, 122.7, 119.9, 118.4, 110.6, 109.4, 75.6, 56.2, 47.6, 44.9, $24.4 \mathrm{ppm}$. IR (film) $\nu_{\max } 1644,1460,1442$, 1346, 1178, 1081, 1015, 962, 906, 727, $698 \mathrm{~cm}^{-1}$. HRMS (ESI) $m / z$ calcd for $\mathrm{C}_{26} \mathrm{H}_{24} \mathrm{~N}_{2} \mathrm{NaO}_{2} 419.1730[\mathrm{M}+\mathrm{Na}]^{+}$, found 419.1732 .

13-Benzyl-6-(methoxymethyl)-4-methyl-6,7,8,13-tetrahydro-5H-benzo[6,7]azocino[5,4-b]indol-5-one (2b). According to procedure $\mathrm{A}$, lactam $\mathbf{2 b}$ was obtained from spiroindoline $\mathbf{1 b}$ (18.9 $\mathrm{mg}, 0.05 \mathrm{mmol})$ and methanol $(1.65 \mathrm{mg}, 0.05 \mathrm{mmol})$ as a pale yellow solid $(17.8 \mathrm{mg}, 87 \%$ yield, eluent: petroleum ether/ethyl acetate $=3 / 1)$. Mp $166-168{ }^{\circ} \mathrm{C} .{ }^{1} \mathrm{H}$ NMR (400 $\left.\mathrm{MHz}, \mathrm{CDCl}_{3}\right) \delta 7.59-7.53(\mathrm{~m}, 1 \mathrm{H}), 7.29-7.17(\mathrm{~m}, 5 \mathrm{H})$, 7.16-7.09 (m, 3H), 7.05-6.98 (m, 3H), $5.23(\mathrm{~d}, J=16.9 \mathrm{~Hz}$, $1 \mathrm{H}), 5.16-5.07(\mathrm{~m}, 2 \mathrm{H}), 4.79(\mathrm{~d}, J=10.4 \mathrm{~Hz}, 1 \mathrm{H}), 3.88-3.79$ $(\mathrm{m}, 1 \mathrm{H}), 3.68-3.47(\mathrm{~m}, 2 \mathrm{H}), 3.19(\mathrm{~s}, 3 \mathrm{H}), 2.91-2.80(\mathrm{~m}$, $1 \mathrm{H}), 2.41(\mathrm{~s}, 3 \mathrm{H}) \mathrm{ppm} .{ }^{13} \mathrm{C} \mathrm{NMR}\left(100 \mathrm{MHz}, \mathrm{CDCl}_{3}\right) \delta 171.6$, $138.1,137.4,137.0,135.5,134.6,130.8,129.2,128.7,128.7$, $128.5,128.0,127.1,126.2,122.5,119.7,118.2,110.7,109.0$, 74.9, 56.3, 47.7, 44.4, 24.0, $19.8 \mathrm{ppm}$. IR (film) $\nu_{\max } 1645$, 1451, 1421, 1349, 1180, 1195, 1078, 1024, 907, 727, $699 \mathrm{~cm}^{-1}$. HRMS (ESI) $m / z$ calcd for $\mathrm{C}_{27} \mathrm{H}_{26} \mathrm{~N}_{2} \mathrm{O}_{2} 433.1886[\mathrm{M}+\mathrm{Na}]^{+}$, found 433.1883 .

13-Benzyl-4-chloro-6-(methoxymethyl)-6,7,8,13-tetrahydro-5H-benzo[6,7]azocino[5,4-b]indol-5-one (2c). According to procedure A, lactam $2 \mathrm{c}$ was obtained from spiroindoline 1c $(20.0 \mathrm{mg}, 0.05 \mathrm{mmol})$ and methanol $(1.65 \mathrm{mg}, 0.05 \mathrm{mmol})$ as a pale yellow solid (16.4 mg, $76 \%$ yield, eluent: petroleum ether/ethyl acetate $=2 / 1)$. Mp $182-184{ }^{\circ} \mathrm{C} .{ }^{1} \mathrm{H}$ NMR (400 $\left.\mathrm{MHz}, \mathrm{CD}_{2} \mathrm{Cl}_{2}\right) \delta 7.60-7.56(\mathrm{~m}, 1 \mathrm{H}), 7.52-7.41(\mathrm{~m}, 3 \mathrm{H})$, 7.29-7.24 (m, 1H), 7.23-7.06 (m, 5H), 6.93-6.83 (m, 2H), $5.22(\mathrm{~d}, J=16.8 \mathrm{~Hz}, 1 \mathrm{H}), 5.11(\mathrm{~d}, J=16.9 \mathrm{~Hz}, 1 \mathrm{H}), 4.97$ (dd, $J=10.2,1.1 \mathrm{~Hz}, 1 \mathrm{H}), 4.86(\mathrm{~d}, J=10.2 \mathrm{~Hz}, 1 \mathrm{H}), 3.86(\mathrm{td}, J=$ 14.2, 4.4 Hz, 1H), 3.67-3.45 (m, 2H), $3.12(\mathrm{~s}, 3 \mathrm{H}), 2.96-$ $2.80(\mathrm{~m}, 1 \mathrm{H}) \mathrm{ppm} .{ }^{13} \mathrm{C}$ NMR $\left(100 \mathrm{MHz}, \mathrm{CD}_{2} \mathrm{Cl}_{2}\right) \delta 172.4$, $138.4,138.1,137.8,134.4,131.0,129.6,129.5,129.3,129.1$, $128.9,128.4,127.4,126.5,122.8,120.1,118.8,110.9,110.1$, 76.0, 56.3, 47.7, 45.3, $24.8 \mathrm{ppm}$. IR (film) $\nu_{\max } 1653,1469$, 1443, 1418, 1360, 1095, 1017, 726, 661, $648 \mathrm{~cm}^{-1}$. HRMS (ESI) $m / z$ calcd for $\mathrm{C}_{26} \mathrm{H}_{23} \mathrm{ClN}_{2} \mathrm{NaO}_{2} 453.1340[\mathrm{M}+\mathrm{Na}]^{+}$, found 453.1337 .

13-Benzyl-6-(methoxymethyl)-3-methyl-6,7,8,13-tetrahydro-5H-benzo[6,7]azocin[5,4-b]indol-5-one (2d). According to procedure A, lactam $2 \mathrm{~d}$ was obtained from spiroindoline $\mathbf{1 d}$ $(18.9 \mathrm{mg}, 0.05 \mathrm{mmol})$ and methanol $(1.65 \mathrm{mg}, 0.05 \mathrm{mmol})$ as a pale yellow solid $(18.5 \mathrm{mg}, 90 \%$ yield, eluent: petroleum ether/ethyl acetate $=3 / 1)$. Mp 73-75 ${ }^{\circ} \mathrm{C} .{ }^{1} \mathrm{H}$ NMR $(400$ $\left.\mathrm{MHz} \mathrm{CDCl}_{3}\right) \delta 7.61-7.52(\mathrm{~m}, 1 \mathrm{H}), 7.35(\mathrm{~d}, J=1.8 \mathrm{~Hz}, 1 \mathrm{H})$, $7.27-7.12(\mathrm{~m}, 7 \mathrm{H}), 7.07(\mathrm{~d}, J=7.9 \mathrm{~Hz}, 1 \mathrm{H}), 7.00-6.95(\mathrm{~m}$, $2 \mathrm{H}), 5.17(\mathrm{~d}, J=16.9 \mathrm{~Hz}, 1 \mathrm{H}), 5.11-4.99(\mathrm{~m}, 2 \mathrm{H}), 4.86(\mathrm{~d}, J$ $=10.3 \mathrm{~Hz}, 1 \mathrm{H}), 3.90(\mathrm{td}, J=14.3,4.6 \mathrm{~Hz}, 1 \mathrm{H}), 3.67-3.46(\mathrm{~m}$, $2 \mathrm{H}), 3.16$ (s, $3 \mathrm{H}), 2.91$ (ddd, $J=16.5,4.4,1.2 \mathrm{~Hz}, 1 \mathrm{H}), 2.39$ (s, 3H) ppm. ${ }^{13} \mathrm{C}$ NMR $\left(100 \mathrm{MHz}, \mathrm{CDCl}_{3}\right) \delta 172.5,139.1$, $138.1,137.5,137.3,134.2$, 130.5, 130.1, 128.7, 128.7, 128.6, $127.1,126.2,126.1,122.5,119.8,118.3,110.6,109.1,75.5$, 56.2, 47.6, 44.8, 24.3, $21.2 \mathrm{ppm}$. IR (film) $\nu_{\max } 1643,1464$, 1440, 1349, 1174, 1092, 1015, 907, 727, $699 \mathrm{~cm}^{-1}$. HRMS (ESI) $m / z$ calcd for $\mathrm{C}_{27} \mathrm{H}_{26} \mathrm{~N}_{2} \mathrm{NaO}_{2} 433.1886[\mathrm{M}+\mathrm{Na}]^{+}$, found 433.1885 .

13-Benzyl-3-chloro-6-(methoxymethyl)-6,7,8,13-tetrahydro-5H-benzo[6,7]azocino[5,4-b]indol-5-one (2e). According to procedure A, lactam 2 a was obtained from spiroindoline $1 \mathbf{e}$ (20.0 $\mathrm{mg}, 0.05 \mathrm{mmol})$ and methanol $(1.65 \mathrm{mg}, 0.05 \mathrm{mmol})$ as a pale yellow solid $(17.5 \mathrm{mg}, 81 \%$ yield, eluent: petroleum ether/ethyl acetate $=3 / 1) . \mathrm{Mp} 65-67{ }^{\circ} \mathrm{C} .{ }^{1} \mathrm{H}$ NMR $(400$ $\left.\mathrm{MHz} \mathrm{CDCl}_{3}\right) \delta 7.60-7.56(\mathrm{~m}, 1 \mathrm{H}), 7.52(\mathrm{~d}, J=2.3 \mathrm{~Hz}, 1 \mathrm{H})$, $7.33(\mathrm{dd}, J=8.3,2.2 \mathrm{~Hz}, 1 \mathrm{H}), 7.28-7.13(\mathrm{~m}, 6 \mathrm{H}), 7.10(\mathrm{~d}, J=$ $8.3 \mathrm{~Hz}, 1 \mathrm{H}), 6.98-6.91(\mathrm{~m}, 2 \mathrm{H}), 5.17(\mathrm{~d}, J=17.0 \mathrm{~Hz}, 1 \mathrm{H})$, 5.09-4.97 (m, 2H), $4.86(\mathrm{~d}, J=10.3 \mathrm{~Hz}, 1 \mathrm{H}), 3.87(\mathrm{td}, J=$ 14.4, $4.3 \mathrm{~Hz}, 1 \mathrm{H}), 3.71-3.48(\mathrm{~m}, 2 \mathrm{H}), 3.14(\mathrm{~s}, 3 \mathrm{H}), 2.92$ (ddd, $J=16.6,4.4,1.3 \mathrm{~Hz}, 1 \mathrm{H}) \mathrm{ppm} .{ }^{13} \mathrm{C} \mathrm{NMR}(100 \mathrm{MHz}$, $\left.\mathrm{CDCl}_{3}\right) \delta 170.8,138.9,137.8,137.8,135.1,132.8,131.9$, $129.4,128.8,128.4,128.3,127.7,127.3,126.0,123.0,120.0$, 
118.5, 110.6, 109.9, 75.6, 56.3, 47.6, 44.9, 24.3 ppm. IR (film) $\nu_{\max } 1651,1464,1441,1350,1180,1093,1015,910,732,698$ $\mathrm{cm}^{-1}$. HRMS (ESI) $\mathrm{m} / z$ calcd for $\mathrm{C}_{26} \mathrm{H}_{23} \mathrm{ClN}_{2} \mathrm{NaO}_{2} 453.1340$ $[\mathrm{M}+\mathrm{Na}]^{+}$, found 453.1338 .

13-Benzyl-3-fluoro-6-(methoxymethyl)-6,7,8,13-tetrahydro-5H-benzo[6,7]azocino[5,4-b]indol-5-one (2f). According to procedure A, lactam $2 \mathbf{f}$ was obtained from spiroindoline $\mathbf{1 f}$ (19.1 mg, $0.05 \mathrm{mmol}$ ) and methanol $(1.65 \mathrm{mg}, 0.05 \mathrm{mmol})$ as a pale yellow solid $(16.4 \mathrm{mg}, 79 \%$ yield, eluent: petroleum ether/ethyl acetate $=3 / 1)$. Mp 76-77 ${ }^{\circ} \mathrm{C} .{ }^{1} \mathrm{H}$ NMR $(400$ $\left.\mathrm{MHz}, \mathrm{CDCl}_{3}\right) \delta 7.61-7.54(\mathrm{~m}, 1 \mathrm{H}), 7.28-7.12(\mathrm{~m}, 8 \mathrm{H}), 7.06$ $(\mathrm{td}, J=8.3,2.7 \mathrm{~Hz}, 1 \mathrm{H}), 6.99-6.92(\mathrm{~m}, 2 \mathrm{H}), 5.17(\mathrm{~d}, J=17.0$ $\mathrm{Hz}, 1 \mathrm{H}), 5.09-4.99(\mathrm{~m}, 2 \mathrm{H}), 4.86(\mathrm{~d}, J=10.3 \mathrm{~Hz}, 1 \mathrm{H}), 3.86$ $(\mathrm{td}, J=14.5,4.4 \mathrm{~Hz}, 1 \mathrm{H}), 3.71-3.48(\mathrm{~m}, 2 \mathrm{H}), 3.14(\mathrm{~s}, 3 \mathrm{H})$, 2.92 (ddd, $J=16.5,4.5,1.3 \mathrm{~Hz}, 1 \mathrm{H}) \mathrm{ppm} .{ }^{13} \mathrm{C} \mathrm{NMR}(100$ $\left.\mathrm{MHz}, \mathrm{CDCl}_{3}\right) \delta 170.87,163.98,161.49,139.46(\mathrm{~d}, J=8.1$ $\mathrm{Hz}), 137.73$ (d, $J=19.2 \mathrm{~Hz}), 132.96,132.57(\mathrm{~d}, J=8.1 \mathrm{~Hz})$, $128.75,128.42,127.26,125.97,125.32$ (d, $J=3.0 \mathrm{~Hz}), 122.82$, $119.96,118.39,116.52(\mathrm{~d}, J=12.1 \mathrm{~Hz}), 115.36(\mathrm{~d}, J=23.2$ $\mathrm{Hz}), 110.55,109.53,75.65,56.27,47.55,44.93,24.27 .{ }^{19} \mathrm{~F}$ NMR (376 MHz, $\left.\mathrm{CDCl}_{3}\right) \delta-111.48$ (s, 1F) ppm. IR (film) $\nu_{\max } 1647,1464,1441,1347,1306,1270,1201,1175,1088$, $1015,962,908,728,699 \mathrm{~cm}^{-1}$. HRMS (ESI) $\mathrm{m} / z$ calcd for $\mathrm{C}_{26} \mathrm{H}_{23} \mathrm{FN}_{2} \mathrm{NaO}_{2} 437.1636\left[\mathrm{M}+\mathrm{Na}^{+}\right.$, found 437.1633 .

13-Benzyl-3-methoxy-6-(methoxymethyl)-6,7,8,13-tetrahydro-5H-benzo[6,7]azocino[5,4-b]indol-5-one (2g). According to procedure $\mathrm{A}$, lactam $2 \mathrm{~g}$ was obtained from spiroindoline $1 \mathrm{~g}(19.7 \mathrm{mg}, 0.05 \mathrm{mmol})$ and methanol $(1.65$ $\mathrm{mg}, 0.05 \mathrm{mmol})$ as a pale yellow solid $(18.8 \mathrm{mg}, 88 \%$ yield, eluent: petroleum ether/ethyl acetate $=3 / 2)$. Mp 66-68 ${ }^{\circ} \mathrm{C}$. ${ }^{1} \mathrm{H} \mathrm{NMR}\left(400 \mathrm{MHz}, \mathrm{CDCl}_{3}\right) \delta 7.61-7.50(\mathrm{~m}, 1 \mathrm{H}), 7.28-7.11$ (m, 6H), 7.10-7.04 (m, 2H), 7.01-6.95 (m, 2H), 6.89 (dd, J $=8.6,2.7 \mathrm{~Hz}, 1 \mathrm{H}), 5.17(\mathrm{~d}, J=17.0 \mathrm{~Hz}, 1 \mathrm{H}), 5.09-4.99(\mathrm{~m}$, $2 \mathrm{H}), 4.85(\mathrm{~d}, J=10.2 \mathrm{~Hz}, 1 \mathrm{H}), 3.91(\mathrm{td}, J=14.5,4.6 \mathrm{~Hz}, 1 \mathrm{H})$, $3.85(\mathrm{~s}, 3 \mathrm{H}), 3.69-3.46(\mathrm{~m}, 2 \mathrm{H}), 3.15(\mathrm{~s}, 3 \mathrm{H}), 2.91$ (ddd, $J=$ 16.4, 4.5, $1.3 \mathrm{~Hz}, 1 \mathrm{H}) \mathrm{ppm} .{ }^{13} \mathrm{C} \mathrm{NMR}\left(100 \mathrm{MHz}, \mathrm{CDCl}_{3}\right) \delta$ $172.1,160.0,138.7,138.1,137.4,134.0,131.9,128.7,128.6$, $127.1,126.1,122.4,121.4,119.7,118.2,115.8,112.7,110.5$, 108.9, 75.6, 56.2, 55.5, 47.5, 45.0, 24.3 ppm. IR (film) $\nu_{\max }$ 1644, 1464, 1452, 1431, 1349, 1315, 1293, 1229, 1182, 1083, $1032,1014,908,727,699 \mathrm{~cm}^{-1}$. HRMS (ESI) $\mathrm{m} / z$ calcd for $\mathrm{C}_{27} \mathrm{H}_{26} \mathrm{~N}_{2} \mathrm{NaO}_{3} 449.1836[\mathrm{M}+\mathrm{Na}]^{+}$, found 449.1833 .

13-Benzyl-2,3-dimethoxy-6-(methoxymethyl)-6,7,8,13-tetrahydro-5H-benzo[6,7]azocino[5,4-b]indol-5-one (2h). According to procedure $\mathrm{A}$, lactam $2 \mathrm{~h}$ was obtained from spiroindoline $1 \mathrm{~h}(21.2 \mathrm{mg}, 0.05 \mathrm{mmol})$ and methanol (1.65 $\mathrm{mg}, 0.05 \mathrm{mmol})$ as a pale yellow solid $(18.5 \mathrm{mg}, 81 \%$ yield, eluent: petroleum ether/ethyl acetate $=1 / 1)$. Mp 89-90 ${ }^{\circ} \mathrm{C}$. ${ }^{1} \mathrm{H}$ NMR $\left(400 \mathrm{MHz}, \mathrm{CDCl}_{3}\right) \delta 7.60(\mathrm{dt}, J=6.9,1.4 \mathrm{~Hz}, 1 \mathrm{H})$, 7.33-7.13 (m, 6H), 7.10-7.00 (m, 3H), $6.53(\mathrm{~s}, 1 \mathrm{H}), 5.21(\mathrm{~d}$, $J=17.5 \mathrm{~Hz}, 1 \mathrm{H}), 5.05(\mathrm{dd}, J=10.1,1.1 \mathrm{~Hz}, 1 \mathrm{H}), 4.96(\mathrm{~d}, J=$ $17.5 \mathrm{~Hz}, 1 \mathrm{H}), 4.82$ (d, $J=10.1 \mathrm{~Hz}, 1 \mathrm{H}), 3.99$ (dd, $J=14.8,4.2$ $\mathrm{Hz}, 1 \mathrm{H}), 3.93$ (s, 3H), 3.70-3.47 (m, 2H), 3.19 (s, 3H), 3.18 (s, 3H), 2.94 (ddd, $J=16.6,4.3,1.4 \mathrm{~Hz}, 1 \mathrm{H}) \mathrm{ppm} .{ }^{13} \mathrm{C}$ NMR $\left(100 \mathrm{MHz}, \mathrm{CDCl}_{3}\right) \delta 172.3,149.3,149.2,138.8,137.9,134.2$, $129.2,128.9,128.4,127.1,125.8,122.7,121.8,119.9,118.4$, $113.1,110.9,110.1,108.9,75.7,56.2,56.1,55.1,47.7,45.0$, $24.2 \mathrm{ppm}$. IR (film) $\nu_{\max } 1641,1604,1515,1465,1429,1350$, 1267, 1249, 1222, 1205, 1167, 1082, 1043, 910, 729, $699 \mathrm{~cm}^{-1}$. HRMS (ESI) $m / z$ calcd for $\mathrm{C}_{28} \mathrm{H}_{28} \mathrm{~N}_{2} \mathrm{NaO}_{4} 479.1941[\mathrm{M}+$ $\mathrm{Na}]^{+}$, found 479.1939 .

13-Benzyl-6-(methoxymethyl)-2-methyl-6,7,8,13-tetrahydro-5H-benzo[6,7]azocino[5,4-b]indol-5-one (2i). According to procedure A, lactam $2 \mathbf{i}$ was obtained from spiroindoline $\mathbf{1 i}$ (18.9 mg, $0.05 \mathrm{mmol}$ ) and methanol ( $1.65 \mathrm{mg}, 0.05 \mathrm{mmol})$ as a pale yellow solid $(17.0 \mathrm{mg}, 83 \%$ yield, eluent: petroleum ether/ethyl acetate $=3 / 1)$. Mp 57-58 ${ }^{\circ} \mathrm{C} .{ }^{1} \mathrm{H}$ NMR (400 $\left.\mathrm{MHz}, \mathrm{CDCl}_{3}\right) \delta 7.62-7.54(\mathrm{~m}, 1 \mathrm{H}), 7.43(\mathrm{~d}, J=7.8 \mathrm{~Hz}, 1 \mathrm{H})$, $7.26-7.11(\mathrm{~m}, 7 \mathrm{H}), 7.02-6.95(\mathrm{~m}, 2 \mathrm{H}), 6.92-6.87(\mathrm{~m}, 1 \mathrm{H})$, $5.20(\mathrm{~d}, J=16.8 \mathrm{~Hz}, 1 \mathrm{H}), 5.06-4.94(\mathrm{~m}, 2 \mathrm{H}), 4.86(\mathrm{~d}, J=$ $10.2 \mathrm{~Hz}, 1 \mathrm{H}), 3.90(\mathrm{td}, J=14.3,4.4 \mathrm{~Hz}, 1 \mathrm{H}), 3.68-3.46(\mathrm{~m}$, $2 \mathrm{H}), 3.14$ (s, 3H), 2.90 (ddd, $J=16.4,4.4,1.2 \mathrm{~Hz}, 1 \mathrm{H}), 2.19$ $(\mathrm{s}, 3 \mathrm{H}) \mathrm{ppm} .{ }^{13} \mathrm{C}$ NMR $\left(100 \mathrm{MHz}, \mathrm{CDCl}_{3}\right) \delta 172.5,139.2$, $138.3,137.8,134.5,134.3,131.4,129.6,129.1,128.6,128.5$, $128.2,127.1,126.2,122.6,119.8,118.4,110.4,109.4,75.6$, 56.1, 47.7, 44.9, 24.3, $21.1 \mathrm{ppm}$. IR (film) $\nu_{\max } 1643,1465$, 1453, 1428, 1349, 11178, 1091, 1022, 908, 828, 727, $707 \mathrm{~cm}^{-1}$. HRMS (ESI) $m / z$ calcd for $\mathrm{C}_{27} \mathrm{H}_{26} \mathrm{~N}_{2} \mathrm{NaO}_{2}$ 433.1886 $[\mathrm{M}+$ $\mathrm{Na}]^{+}$, found 433.1885 .

13-Benzyl-2-fluoro-6-(methoxymethyl)-6,7,8,13-tetrahydro-5H-benzo[6,7]azocino[5,4-b]indol-5-one (2j). According to procedure $A$, lactam $2 \mathbf{j}$ was obtained from spiroindoline $\mathbf{1 j}$ (19.1 mg, $0.05 \mathrm{mmol})$ and methanol $(1.65 \mathrm{mg}, 0.05 \mathrm{mmol})$ as a pale yellow solid $(16.6 \mathrm{mg}, 80 \%$ yield, eluent: petroleum ether/ethyl acetate $=3 / 1)$. Mp 194-196 ${ }^{\circ} \mathrm{C} .{ }^{1} \mathrm{H}$ NMR (400 $\left.\mathrm{MHz}, \mathrm{CDCl}_{3}\right) \delta 7.59(\mathrm{dd}, J=7.6,1.3 \mathrm{~Hz}, 1 \mathrm{H}), 7.52(\mathrm{dd}, J=$ 8.5, 5.7 Hz, 1H), 7.29-7.09 (m, 7H), 6.98-6.91 (m, 2H), 6.87 (dd, $J=9.5,2.6 \mathrm{~Hz}, 1 \mathrm{H}), 5.20(\mathrm{~d}, J=16.9 \mathrm{~Hz}, 1 \mathrm{H}), 5.07$ (d, $J$ $=16.9 \mathrm{~Hz}, 1 \mathrm{H}), 5.00(\mathrm{dd}, J=10.3,1.0 \mathrm{~Hz}, 1 \mathrm{H}), 4.88(\mathrm{~d}, J=$ $10.3 \mathrm{~Hz}, 1 \mathrm{H}), 3.87$ (td, $J=14.3,4.4 \mathrm{~Hz}, 1 \mathrm{H}), 3.68-3.49(\mathrm{~m}$, $2 \mathrm{H}$ ), 3.13 (s, 3H), 2.93 (ddd, $J=16.5,4.3,1.2 \mathrm{~Hz}, 1 \mathrm{H}) \mathrm{ppm}$. ${ }^{13} \mathrm{C}$ NMR $\left(100 \mathrm{MHz}, \mathrm{CDCl}_{3}\right) \delta 171.45,163.85,161.36$, $137.81,137.63,133.63(\mathrm{~d}, J=3.0 \mathrm{~Hz}), 132.67(\mathrm{~d}, J=2.0 \mathrm{~Hz})$, $131.65(\mathrm{~d}, J=8.1 \mathrm{~Hz}), 130.36(\mathrm{~d}, J=9.1 \mathrm{~Hz}), 128.77,128.37$, $127.34,126.02,123.08,120.05,118.56,117.46(\mathrm{~d}, J=22.2$ $\mathrm{Hz}), 116.12(\mathrm{~d}, J=22.2 \mathrm{~Hz}), 110.62,110.19,75.74,56.22$, 47.70, 44.91, $24.36 \mathrm{ppm} .{ }^{19} \mathrm{~F}$ NMR $\left(376 \mathrm{MHz}, \mathrm{CDCl}_{3}\right) \delta$ $-110.52(\mathrm{td}, J=8.7,5.8 \mathrm{~Hz}, 1 \mathrm{~F}) \mathrm{ppm}$. IR (film) $\nu_{\max } 1647$, 1606, 1465, 1453, 1431, 1346, 1179, 1090, 1021, 909, 729, 699 $\mathrm{cm}^{-1}$. HRMS (ESI) $\mathrm{m} / z$ calcd for $\mathrm{C}_{26} \mathrm{H}_{23} \mathrm{FN}_{2} \mathrm{NaO}_{2} 437.1636$ $[\mathrm{M}+\mathrm{Na}]^{+}$, found 437.1634 .

13-Benzyl-2-methoxy-6-(methoxymethyl)-6,7,8,13-tetrahydro-5H-benzo[6,7]azocino[5,4-b]indol-5-one (2k). According to procedure $\mathrm{A}$, lactam $2 \mathrm{k}$ was obtained from spiroindoline $1 \mathrm{k}(19.7 \mathrm{mg}, 0.05 \mathrm{mmol})$ and methanol $(1.65 \mathrm{mg}, 0.05 \mathrm{mmol})$ as a pale yellow solid $(18.3 \mathrm{mg}, 86 \%$ yield, eluent: petroleum ether/ethyl acetate $=3 / 1) . \mathrm{Mp} 67-69{ }^{\circ} \mathrm{C} .{ }^{1} \mathrm{H}$ NMR $(400$ $\left.\mathrm{MHz}_{\mathrm{CDCl}}\right) \delta 7.60(\mathrm{dt}, J=7.1,1.4 \mathrm{~Hz}, 1 \mathrm{H}), 7.46(\mathrm{~d}, J=8.6$ $\mathrm{Hz}, 1 \mathrm{H}), 7.30-7.13(\mathrm{~m}, 6 \mathrm{H}), 7.06-6.99(\mathrm{~m}, 2 \mathrm{H}), 6.95$ (dd, $J$ $=8.6,2.6 \mathrm{~Hz}, 1 \mathrm{H}), 6.62(\mathrm{~d}, J=2.5 \mathrm{~Hz}, 1 \mathrm{H}), 5.21(\mathrm{~d}, J=17.2$ $\mathrm{Hz}, 1 \mathrm{H}), 5.07-4.97(\mathrm{~m}, 2 \mathrm{H}), 4.85(\mathrm{~d}, J=10.3 \mathrm{~Hz}, 1 \mathrm{H}), 3.98-$ $3.87(\mathrm{~m}, 1 \mathrm{H}), 3.67-3.48(\mathrm{~m}, 2 \mathrm{H}), 3.34(\mathrm{~s}, 3 \mathrm{H}), 3.16(\mathrm{~s}, 3 \mathrm{H})$, 2.93 (ddd, $J=16.5,4.4,1.2 \mathrm{~Hz}, 1 \mathrm{H})$ ppm. ${ }^{13} \mathrm{C}$ NMR $(100$ $\left.\mathrm{MHz}, \mathrm{CDCl}_{3}\right) \delta 172.4,159.9,138.5,137.9,134.1,130.7,129.9$, $129.5,128.8,128.4,127.1,125.9,122.8,119.9,118.5,116.0$, $114.5,110.4,109.5,75.6,56.1,54.8,47.6,44.8,24.3$ ppm. IR (film) $\nu_{\max } 1639,1602,1465,1431,1348,1307,1239,1121$, 1177, 1090, 1048, 1022, 907, 727, $698 \mathrm{~cm}^{-1}$. HRMS (ESI) $\mathrm{m} /$ $z$ calcd for $\mathrm{C}_{27} \mathrm{H}_{26} \mathrm{~N}_{2} \mathrm{NaO}_{3} 449.1836[\mathrm{M}+\mathrm{Na}]^{+}$, found 449.1834.

13-Benzyl-6-(methoxymethyl)-10-methyl-6,7,8,13-tetrahydro-5H-benzo[6,7]azocino[5,4-b]indol-5-one (2I). According to procedure $A$, lactam 21 was obtained from spiroindoline 11 (19.1 mg, $0.05 \mathrm{mmol})$ and methanol $(1.65 \mathrm{mg}, 0.05 \mathrm{mmol})$ as a pale yellow solid $(17.6 \mathrm{mg}, 86 \%$ yield, eluent: petroleum ether/ethyl acetate $=3 / 1)$. Mp 62-64 ${ }^{\circ} \mathrm{C} .{ }^{1} \mathrm{H}$ NMR $(400$ 
$\left.\mathrm{MHz}, \mathrm{CDCl}_{3}\right) \delta 7.56-7.50(\mathrm{~m}, 1 \mathrm{H}), 7.43(\mathrm{td}, J=7.5,1.3 \mathrm{~Hz}$, $1 \mathrm{H}), 7.39-7.31(\mathrm{~m}, 2 \mathrm{H}), 7.27-7.14(\mathrm{~m}, 4 \mathrm{H}), 7.07-6.92(\mathrm{~m}$, $4 \mathrm{H}), 5.14(\mathrm{~d}, J=16.9 \mathrm{~Hz}, 1 \mathrm{H}), 5.08-4.99(\mathrm{~m}, 2 \mathrm{H}), 4.86(\mathrm{~d}, J$ $=10.2 \mathrm{~Hz}, 1 \mathrm{H}), 3.96-3.79(\mathrm{~m}, 1 \mathrm{H}), 3.68-3.43(\mathrm{~m}, 2 \mathrm{H}), 3.17$ (s, 3H), 2.89 (ddd, $J=16.5,4.4,1.3 \mathrm{~Hz}, 1 \mathrm{H}), 2.46(\mathrm{~s}, 3 \mathrm{H})$ ppm. ${ }^{13} \mathrm{C} \mathrm{NMR}\left(100 \mathrm{MHz}, \mathrm{CDCl}_{3}\right) \delta 172.4,138.1,137.4$, 136.0, 134.1, 130.5, 129.4, 129.2, 129.1, 128.9, 128.8, 128.7, 128.2, 127.1, 126.1, 124.2, 118.1, 110.3, 108.9, 75.5, 56.2, 47.7, 44.8, 24.3, $21.5 \mathrm{ppm}$. IR (film) $\nu_{\max } 1644,1466,1442,1420$, 1349, 1309, 1180, 1091, 1036, 1014, 907, 790, 774, 727, 699 $\mathrm{cm}^{-1}$. HRMS (ESI) $\mathrm{m} / z$ calcd for $\mathrm{C}_{27} \mathrm{H}_{26} \mathrm{~N}_{2} \mathrm{NaO}_{2} 433.1886$ $[\mathrm{M}+\mathrm{Na}]^{+}$, found 433.1884 .

13-Benzyl-10-chloro-6-(methoxymethyl)-6,7,8,13-tetrahydro-5H-benzo[6,7]azocino[5,4-b]indol-5-one (2m). According to procedure A, lactam $2 \mathrm{~m}$ was obtained from spiroindoline $1 \mathrm{~m}(20.0 \mathrm{mg}, 0.05 \mathrm{mmol})$ and methanol $(1.65 \mathrm{mg}, 0.05$ $\mathrm{mmol})$ as a pale yellow solid $(15.7 \mathrm{mg}, 73 \%$ yield, eluent: petroleum ether/ethyl acetate $=2 / 1)$. Mp $63-65{ }^{\circ} \mathrm{C} .{ }^{1} \mathrm{H}$ $\operatorname{NMR}\left(400 \mathrm{MHz}, \mathrm{CDCl}_{3}\right) \delta 7.54(\mathrm{dt}, J=4.4,1.8 \mathrm{~Hz}, 2 \mathrm{H}), 7.47$ $(\mathrm{td}, J=7.5,1.3 \mathrm{~Hz}, 1 \mathrm{H}), 7.38(\mathrm{td}, J=7.5,1.5 \mathrm{~Hz}, 1 \mathrm{H}), 7.28-$ $7.16(\mathrm{~m}, 4 \mathrm{H}), 7.11(\mathrm{dd}, J=8.7,2.0 \mathrm{~Hz}, 1 \mathrm{H}), 7.05(\mathrm{~d}, J=8.7$ $\mathrm{Hz}, 1 \mathrm{H}), 6.93(\mathrm{dd}, J=7.6,1.8 \mathrm{~Hz}, 2 \mathrm{H}), 5.15(\mathrm{~d}, J=16.9 \mathrm{~Hz}$, $1 \mathrm{H}), 5.09-4.96(\mathrm{~m}, 2 \mathrm{H}), 4.91(\mathrm{~d}, J=10.3 \mathrm{~Hz}, 1 \mathrm{H}), 3.86(\mathrm{td}, J$ $=14.5,4.4 \mathrm{~Hz}, 1 \mathrm{H}), 3.62(\mathrm{ddd}, J=15.0,6.1,1.4 \mathrm{~Hz}, 1 \mathrm{H}), 3.47$ (ddd, $J=16.5,13.9,6.2 \mathrm{~Hz}, 1 \mathrm{H}), 3.14(\mathrm{~s}, 3 \mathrm{H}), 2.88$ (ddd, $J=$ 16.5, 4.5, $1.3 \mathrm{~Hz}, 1 \mathrm{H})$ ppm. ${ }^{13} \mathrm{C} \mathrm{NMR}\left(100 \mathrm{MHz}, \mathrm{CDCl}_{3}\right) \delta$ $172.2,137.5,137.5,135.9,135.4,130.4,129.6,129.4,129.3$, $128.8,128.7,128.2,127.3,126.0,125.6,122.9,117.9,111.7$, 109.1, 75.7, 56.3, 47.7, 45.0, 24.4 ppm. IR (film) $\nu_{\max } 1644$, 1466, 1440, 1420, 1179, 1091, 1036, 1013, 908, 729, $700 \mathrm{~cm}^{-1}$. HRMS (ESI) $m / z$ calcd for $\mathrm{C}_{26} \mathrm{H}_{23} \mathrm{ClN}_{2} \mathrm{NaO}_{2} 453.1340[\mathrm{M}+$ $\mathrm{Na}]^{+}$, found 453.1340 .

13-Benzyl-10-methoxy-6-(methoxymethyl)-6,7,8, 13-tetrahydro-5H-benzo[6,7]azocino[5,4-b]indol-5-one (2n). According to procedure $\mathrm{A}$, lactam $\mathbf{2 n}$ was obtained from spiroindoline 1n $(19.7 \mathrm{mg}, 0.05 \mathrm{mmol})$ and methanol (1.65 $\mathrm{mg}, 0.05 \mathrm{mmol})$ as a pale yellow solid $(17.7 \mathrm{mg}, 83 \%$ yield, eluent: petroleum ether/ethyl acetate $=3 / 1)$. Mp 60-62 ${ }^{\circ} \mathrm{C}$. ${ }^{1} \mathrm{H}$ NMR (400 MHz, $\left.\mathrm{CDCl}_{3}\right) \delta 7.56-7.49(\mathrm{~m}, 1 \mathrm{H}), 7.44(\mathrm{td}, J$ $=7.5,1.3 \mathrm{~Hz}, 1 \mathrm{H}), 7.37(\mathrm{td}, J=7.5,1.5 \mathrm{~Hz}, 1 \mathrm{H}), 7.28-7.15$ (m, 4H), 7.06-6.99 (m, 2H), 6.99-6.93 (m, 2H), 6.83 (dd, $J$ $=8.8,2.4 \mathrm{~Hz}, 1 \mathrm{H}), 5.13(\mathrm{~d}, J=16.9 \mathrm{~Hz}, 1 \mathrm{H}), 5.07-4.99(\mathrm{~m}$, $2 \mathrm{H}), 4.91(\mathrm{~d}, J=10.3 \mathrm{~Hz}, 1 \mathrm{H}), 3.93-3.84(\mathrm{~m}, 4 \mathrm{H}), 3.62$ (ddd, $J=15.0,6.1,1.4 \mathrm{~Hz}, 1 \mathrm{H}), 3.49(\mathrm{ddd}, J=16.4,13.9,6.1 \mathrm{~Hz}$, $1 \mathrm{H}), 3.16$ (s, 3H), 2.89 (ddd, $J=16.5,4.4,1.4 \mathrm{~Hz}, 1 \mathrm{H}) \mathrm{ppm}$. ${ }^{13} \mathrm{C}$ NMR $\left(100 \mathrm{MHz}, \mathrm{CDCl}_{3}\right) \delta 172.3,154.4,138.0,137.4$, $134.7,132.9,130.5,129.3,129.3,128.9,128.7,128.2,127.1$, 126.1, 112.6, 111.4, 109.0, 100.3, 75.7, 56.2, 55.9, 47.7, 44.9, $24.5 \mathrm{ppm}$. IR (film) $\nu_{\max } 1647,1483,1466,1442,1291,1208$, 1172, 1091, 1046, 1028, 909, 774, 729, $700 \mathrm{~cm}^{-1}$. HRMS (ESI) $m / z$ calcd for $\mathrm{C}_{27} \mathrm{H}_{26} \mathrm{~N}_{2} \mathrm{NaO}_{3} 449.1836[\mathrm{M}+\mathrm{Na}]^{+}$, found 449.1833 .

6-(Methoxymethyl)-13-methyl-6,7,8,13-tetrahydro-5Hbenzo[6,7]azocino[5,4-b]indol-5-one (2o). According to procedure A, lactam 20 was obtained from spiroindoline 10 (14.4 mg, $0.05 \mathrm{mmol}$ ) and methanol ( $1.65 \mathrm{mg}, 0.05 \mathrm{mmol}$ ) as a pale yellow solid $(14.6 \mathrm{mg}, 85 \%$ yield, eluent: petroleum ether/ethyl acetate $=2 / 1)$. Mp 55-57 ${ }^{\circ} \mathrm{C} .{ }^{1} \mathrm{H}$ NMR $(400$ $\left.\mathrm{MHz} \mathrm{CDCl}_{3}\right) \delta 7.60-7.45(\mathrm{~m}, 4 \mathrm{H}), 7.34-7.23(\mathrm{~m}, 3 \mathrm{H}), 7.15$ (ddd, $J=7.9,6.7,1.3 \mathrm{~Hz}, 1 \mathrm{H}), 5.07(\mathrm{dd}, J=10.2,1.2 \mathrm{~Hz}, 1 \mathrm{H})$, $4.78(\mathrm{~d}, J=10.2 \mathrm{~Hz}, 1 \mathrm{H}), 3.90-3.82(\mathrm{~m}, 1 \mathrm{H}), 3.62$ (ddd, $J=$ 15.0, 6.0, $1.5 \mathrm{~Hz}, 1 \mathrm{H}), 3.55-3.45(\mathrm{~m}, 4 \mathrm{H}), 3.19$ (s, 3H), 2.88 (ddd, $J=16.6,4.3,1.4 \mathrm{~Hz}, 1 \mathrm{H}) \mathrm{ppm} .{ }^{13} \mathrm{C}$ NMR $(100 \mathrm{MHz}$,
$\left.\mathrm{CDCl}_{3}\right) \delta 172.4,138.0,137.2,133.8,131.0,129.4,129.3$, $128.8,128.2,128.1,122.5,119.5,118.3,109.5,108.8,75.3$, 56.2, 44.6, 31.0, $24.1 \mathrm{ppm}$. IR (film) $\nu_{\max } 1644,1467,1444$, 1421, 1359, 1089, 1036, 1011, 910, 737, $648 \mathrm{~cm}^{-1}$. HRMS (ESI) $m / z$ calcd for $\mathrm{C}_{20} \mathrm{H}_{20} \mathrm{~N}_{2} \mathrm{NaO}_{2} 343.1417[\mathrm{M}+\mathrm{Na}]^{+}$, found 343.1415 .

13-Benzyl-6-(ethoxymethyl)-6,7,8,13-tetrahydro-5Hbenzo[6,7]azocino[5,4-b]indol-5-one (2p). According to procedure A, lactam $2 \mathbf{p}$ was obtained from spiroindoline $\mathbf{1 a}$ $(18.2 \mathrm{mg}, 0.05 \mathrm{mmol})$ and ethanol $(2.3 \mathrm{mg}, 0.05 \mathrm{mmol})$ as a pale yellow solid ( $15.3 \mathrm{mg}$, $74 \%$ yield, eluent: petroleum ether/ ethyl acetate $=3 / 1) . \mathrm{Mp} \mathrm{56-58}{ }^{\circ} \mathrm{C} .{ }^{1} \mathrm{H}$ NMR $(400 \mathrm{MHz}$, $\left.\mathrm{CDCl}_{3}\right) \delta 7.61-7.56(\mathrm{~m}, 1 \mathrm{H}), 7.53(\mathrm{dd}, J=7.6,1.5 \mathrm{~Hz}, 1 \mathrm{H})$, $7.44(\mathrm{td}, J=7.5,1.3 \mathrm{~Hz}, 1 \mathrm{H}), 7.36(\mathrm{td}, J=7.6,1.5 \mathrm{~Hz}, 1 \mathrm{H})$, $7.27-7.10(\mathrm{~m}, 7 \mathrm{H}), 6.99-6.92(\mathrm{~m}, 2 \mathrm{H}), 5.17(\mathrm{~d}, J=16.9 \mathrm{~Hz}$, $1 \mathrm{H}), 5.13-5.02(\mathrm{~m}, 2 \mathrm{H}), 4.96(\mathrm{~d}, J=10.5 \mathrm{~Hz}, 1 \mathrm{H}), 3.89(\mathrm{td}, J$ $=14.3,4.4 \mathrm{~Hz}, 1 \mathrm{H}), 3.70-3.50(\mathrm{~m}, 2 \mathrm{H}), 3.41(\mathrm{dq}, J=9.5,7.0$ $\mathrm{Hz}, 1 \mathrm{H}), 3.27$ (dq, $J=9.5,7.0 \mathrm{~Hz}, 1 \mathrm{H}), 2.92$ (ddd, $J=16.5$, 4.4, $1.2 \mathrm{~Hz}, 1 \mathrm{H}), 1.00(\mathrm{t}, J=7.0 \mathrm{~Hz}, 3 \mathrm{H}) \mathrm{ppm} .{ }^{13} \mathrm{C}$ NMR $(100$ $\left.\mathrm{MHz}, \mathrm{CDCl}_{3}\right) \delta 172.2$, 138.0, 137.6, 137.5, 134.0, 130.6, 129.2, $128.9,128.7,128.5,128.1,127.1,126.1,122.7,119.8,118.4$, $110.6,109.5,74.2,64.2$, 47.6, 45.0, 24.4, 15.0 ppm. IR (film) $\nu_{\max } 2973,2925,1644,1464,1443,1420,1386,1349,1319$, $1182,1089,1036,1014,909,729,698 \mathrm{~cm}^{-1}$. HRMS (ESI) $\mathrm{m} /$ $z$ calcd for $\mathrm{C}_{27} \mathrm{H}_{26} \mathrm{~N}_{2} \mathrm{NaO}_{2} 433.1886[\mathrm{M}+\mathrm{Na}]^{+}$, found 433.1890.

13-Benzyl-6-((benzyloxy)methyl)-6,7,8,13-tetrahydro-5Hbenzo[6,7]azocino[5,4-b]indol-5-one (2q). According to procedure A, lactam $\mathbf{2 q}$ was obtained from spiroindoline $1 \mathrm{a}$ (18.2 $\mathrm{mg}, 0.05 \mathrm{mmol}$ ) and benzyl alcohol $(5.4 \mathrm{mg}, 0.05 \mathrm{mmol})$ as a pale yellow solid $(19.5 \mathrm{mg}, 83 \%$ yield, eluent: petroleum ether/ethyl acetate $=2 / 1)$. Mp 61-63 ${ }^{\circ} \mathrm{C} .{ }^{1} \mathrm{H}$ NMR $(400$ $\left.\mathrm{MHz}, \mathrm{CDCl}_{3}\right) \delta 7.51(\mathrm{dd}, J=7.7,1.4 \mathrm{~Hz}, 2 \mathrm{H}), 7.44(\mathrm{td}, J=$ 7.5, $1.3 \mathrm{~Hz}, 1 \mathrm{H}), 7.41-7.34(\mathrm{~m}, 1 \mathrm{H}), 7.27-7.14(\mathrm{~m}, 9 \mathrm{H})$, 7.14-7.03 (m, 3H), 7.00-6.91 (m, 2H), 5.25-5.00 (m, 4H), $4.39(\mathrm{~d}, J=11.6 \mathrm{~Hz}, 1 \mathrm{H}), 4.28(\mathrm{~d}, J=11.6 \mathrm{~Hz}, 1 \mathrm{H}), 3.91(\mathrm{td}, J$ $=14.4,4.4 \mathrm{~Hz}, 1 \mathrm{H}), 3.73-3.48(\mathrm{~m}, 2 \mathrm{H}), 2.92(\mathrm{ddd}, J=16.5$, 4.4, $1.3 \mathrm{~Hz}, 1 \mathrm{H}) \mathrm{ppm} .{ }^{13} \mathrm{C}$ NMR $\left(100 \mathrm{MHz}, \mathrm{CDCl}_{3}\right) \delta 172.3$, $137.9,137.8,137.6,137.4,134.0,130.6,129.3,129.3,128.9$, $128.7,128.5,128.3,128.2,127.9,127.6,127.2,126.1,122.7$, $119.9,118.6,110.63,109.6,74.6,71.1,65.4,47.7,45.4,24.5$ ppm. IR (film) $\nu_{\max } 2923,1645,1464,1442,1420,1378,1349$, $1319,1071,1017,908,728,696 \mathrm{~cm}^{-1}$. HRMS (ESI) $\mathrm{m} / z$ calcd for $\mathrm{C}_{32} \mathrm{H}_{28} \mathrm{~N}_{2} \mathrm{NaO}_{2} 495.2043[\mathrm{M}+\mathrm{Na}]^{+}$, found 495.2045 .

13-Benzyl-6-(isopropoxymethyl)-6,7,8,13-tetrahydro-5Hbenzo[6,7]azocino[5,4-b]indol-5-one (2r). According to procedure A, lactam $2 \mathbf{r}$ was obtained from spiroindoline $\mathbf{1 a}$ (18.2 $\mathrm{mg}, 0.05 \mathrm{mmol})$ and 2-propanol $(3.0 \mathrm{mg}, 0.05 \mathrm{mmol})$ as a pale yellow solid $(16.3 \mathrm{mg}, 77 \%$ yield, eluent: petroleum ether/ethyl acetate $=3 / 1)$. Mp 67-69 ${ }^{\circ} \mathrm{C} .{ }^{1} \mathrm{H}$ NMR (400 $\left.\mathrm{MHz}, \mathrm{CDCl}_{3}\right) \delta 7.57(\mathrm{dt}, J=6.8,1.4 \mathrm{~Hz}, 1 \mathrm{H}), 7.52(\mathrm{dd}, J=$ $7.6,1.5 \mathrm{~Hz}, 1 \mathrm{H}), 7.44(\mathrm{td}, J=7.5,1.3 \mathrm{~Hz}, 1 \mathrm{H}), 7.36(\mathrm{td}, J=$ 7.6, $1.5 \mathrm{~Hz}, 1 \mathrm{H}), 7.28-7.10(\mathrm{~m}, 7 \mathrm{H}), 7.00-6.88(\mathrm{~m}, 2 \mathrm{H})$, $5.22-4.99(\mathrm{~m}, 2 \mathrm{H}), 4.84(\mathrm{~d}, J=10.6 \mathrm{~Hz}, 1 \mathrm{H}), 3.94-3.79(\mathrm{~m}$, $1 \mathrm{H}), 3.77-3.44(\mathrm{~m}, 3 \mathrm{H}), 2.92(\mathrm{ddd}, J=16.5,4.2,1.5 \mathrm{~Hz}, 1 \mathrm{H})$, $1.15(\mathrm{~d}, J=6.1 \mathrm{~Hz}, 3 \mathrm{H}), 1.00(\mathrm{~d}, J=6.1 \mathrm{~Hz}, 3 \mathrm{H}) \mathrm{ppm} .{ }^{13} \mathrm{C}$ NMR $\left(100 \mathrm{MHz}, \mathrm{CDCl}_{3}\right) \delta 171.9,138.0,137.6,137.5,134.0$, $130.6,129.2,128.9,128.6,128.5,128.1,127.1,126.1,122.6$, $119.7,118.5,110.6,109.5,71.7,69.4,47.6,44.4,24.1,22.3$, $22.2 \mathrm{ppm}$. IR (film) $\nu_{\max } 2970,2924,1644,1464,1443,1420$, $1367,1180,1065,1036,1015,908,731,699 \mathrm{~cm}^{-1}$. HRMS (ESI) $m / z$ calcd for $\mathrm{C}_{28} \mathrm{H}_{28} \mathrm{~N}_{2} \mathrm{NaO}_{2} 447.2043[\mathrm{M}+\mathrm{Na}]^{+}$, found 447.2046. 
13-Benzyl-6-((p-tolylthio)methyl)-6,7,8,13-tetrahydro-5Hbenzo[6,7]azocino[5,4-b]indol-5-one (2s). According to procedure A, lactam $2 \mathrm{~s}$ was obtained from spiroindoline 1a (18.2 mg, $0.05 \mathrm{mmol}$ ) and methyl p-tolyl sulfide (6.9 mg, 0.05 $\mathrm{mmol})$ as a pale yellow solid $(21.0 \mathrm{mg}, 87 \%$ yield, eluent: petroleum ether/ethyl acetate $=1 / 1)$. Mp 67-69 ${ }^{\circ} \mathrm{C} .{ }^{1} \mathrm{H}$ NMR (400 MHz, $\left.\mathrm{CDCl}_{3}\right) \delta 7.59-7.50(\mathrm{~m}, 1 \mathrm{H}), 7.40-7.27$ (m, 4H), 7.26-7.04 (m, 10H), 6.99-6.92 (m, 2H), $5.61(\mathrm{dd}, J$ $=13.4,1.1 \mathrm{~Hz}, 1 \mathrm{H}), 5.13(\mathrm{~d}, J=17.0 \mathrm{~Hz}, 1 \mathrm{H}), 5.00(\mathrm{~d}, J=$ $17.0 \mathrm{~Hz}, 1 \mathrm{H}), 4.21(\mathrm{~d}, J=13.3 \mathrm{~Hz}, 1 \mathrm{H}), 3.89-3.71(\mathrm{~m}, 2 \mathrm{H})$, 3.45 (ddd, $J=16.7,12.3,7.5 \mathrm{~Hz}, 1 \mathrm{H}$ ), 2.88 (ddd, $J=16.7$, 3.7, $2.0 \mathrm{~Hz}, 1 \mathrm{H}), 2.33$ (s, 3H) ppm. ${ }^{13} \mathrm{C}$ NMR $\left(100 \mathrm{MHz}, \mathrm{CDCl}_{3}\right)$ $\delta 170.9,137.9,137.6,137.6,137.2,134.0,132.5,130.4,129.8$, $129.2,129.1,128.8,128.7,128.5,128.0,127.1,126.1,122.7$, $119.8,118.4,110.7,109.0,48.6,47.6,43.9,23.3,21.2$ ppm. IR (film) $\nu_{\max } 2919,1640,1493,1464,1443,1419,1349,1274$, 1249, 1182, 1142, 1022, 908, 870, 730, $698 \mathrm{~cm}^{-1}$. HRMS (ESI) $m / z$ calcd for $\mathrm{C}_{32} \mathrm{H}_{28} \mathrm{~N}_{2} \mathrm{NaOS} 511.1815[\mathrm{M}+\mathrm{Na}]^{+}$, found 511.1816

13-Benzyl-6-((2-hydroxynaphthalen-1-yl)methyl)6,7,8,13-tetrahydro-5H-benzo[6,7]azocino[5,4-b]indol-5one (2t). According to procedure A, lactam $2 \mathrm{t}$ was obtained from spiroindoline $1 \mathrm{a}(18.2 \mathrm{mg}, 0.05 \mathrm{mmol})$ and 2hydroxynaphthalene $(7.2 \mathrm{mg}, 0.05 \mathrm{mmol})$ as a white solid (20.8 mg, $82 \%$ yield, eluent: petroleum ether/ethyl acetate $=$ 1/2). Mp 231-233 ${ }^{\circ} \mathrm{C} .{ }^{1} \mathrm{H}$ NMR (400 MHz, $\left.\mathrm{CDCl}_{3}\right) \delta 10.20$ (s, $1 \mathrm{H}), 8.07(\mathrm{~d}, J=8.6 \mathrm{~Hz}, 1 \mathrm{H}), 7.81(\mathrm{dd}, J=8.2,1.3 \mathrm{~Hz}$, $1 \mathrm{H}), 7.72(\mathrm{~d}, J=8.9 \mathrm{~Hz}, 1 \mathrm{H}), 7.65-7.54(\mathrm{~m}, 2 \mathrm{H}), 7.53-7.47$ (m, $1 \mathrm{H}), 7.46-7.32(\mathrm{~m}, 3 \mathrm{H}), 7.30-7.11(\mathrm{~m}, 8 \mathrm{H}), 7.01-6.90$ $(\mathrm{m}, 2 \mathrm{H}), 5.20(\mathrm{dd}, J=16.2,12.2 \mathrm{~Hz}, 2 \mathrm{H}), 5.05(\mathrm{~d}, J=17.0 \mathrm{~Hz}$, $1 \mathrm{H}), 4.85(\mathrm{~d}, J=15.4 \mathrm{~Hz}, 1 \mathrm{H}), 3.93-3.54(\mathrm{~m}, 3 \mathrm{H}), 2.99$ (dd, $J$ $=15.7,3.8 \mathrm{~Hz}, 1 \mathrm{H}) \mathrm{ppm} .{ }^{13} \mathrm{C} \operatorname{NMR}\left(100 \mathrm{MHz}, \mathrm{CDCl}_{3}\right) \delta$ $173.7,155.7,137.9,137.7,135.7,134.0,133.9,130.7,130.6$, $129.9,129.7,129.2,129.0,128.9,128.8,128.2,127.2,127.2$, $126.0,122.9,122.7,120.8,120.0,119.9,118.3,113.0,110.7$, 108.7, 47.7, 46.0, 38.5, $23.1 \mathrm{ppm}$. IR (film) $\nu_{\max } 3067,1598$, $1582,1514,1465,1452,1438,1360,1348,1317,1294,1273$, $1212,1182,1042,815,758,747,729 \mathrm{~cm}^{-1}$. HRMS (ESI) $\mathrm{m} / z$ calcd for $\mathrm{C}_{35} \mathrm{H}_{28} \mathrm{~N}_{2} \mathrm{NaO}_{2}$ 531.2043 $[\mathrm{M}+\mathrm{Na}]^{+}$, found 531.2042 .

6-((1H-Indol-3-yl)methyl)-13-benzyl-6,7,8, 13-tetrahydro$5 \mathrm{H}$-benzo[6,7]azocino[5,4-b]indol-5-one (2u). According to procedure $A$, lactam $\mathbf{2} \mathbf{u}$ was obtained from spiroindoline $\mathbf{1 a}$ $(18.2 \mathrm{mg}, 0.05 \mathrm{mmol})$ and indole $(5.9 \mathrm{mg}, 0.05 \mathrm{mmol})$ as a white solid (16.8 mg, 70\% yield, eluent: petroleum ether/ethyl acetate $=1 / 2) . \mathrm{Mp} 164-166{ }^{\circ} \mathrm{C} .{ }^{1} \mathrm{H}$ NMR $(400 \mathrm{MHz}$, $\left.\mathrm{CDCl}_{3}\right) \delta 8.27-8.14(\mathrm{~m}, 1 \mathrm{H}), 7.77(\mathrm{dd}, J=7.9,1.2 \mathrm{~Hz}, 1 \mathrm{H})$, $7.57(\mathrm{dd}, J=7.7,1.4 \mathrm{~Hz}, 1 \mathrm{H}), 7.47-6.86(\mathrm{~m}, 16 \mathrm{H}), 5.35(\mathrm{~d}, J$ $=14.8 \mathrm{~Hz}, 1 \mathrm{H}), 5.15(\mathrm{~d}, J=17.0 \mathrm{~Hz}, 1 \mathrm{H}), 5.04(\mathrm{~d}, J=17.0$ $\mathrm{Hz}, 1 \mathrm{H}), 4.37$ (d, $J=14.8 \mathrm{~Hz}, 1 \mathrm{H}), 3.84-3.65(\mathrm{~m}, 1 \mathrm{H}), 3.52-$ $3.28(\mathrm{~m}, 2 \mathrm{H}), 2.87-2.68(\mathrm{~m}, 1 \mathrm{H}) \mathrm{ppm} .{ }^{13} \mathrm{C} \mathrm{NMR}(100 \mathrm{MHz}$, $\left.\mathrm{CDCl}_{3}\right) \delta 170.9,138.1,138.1,137.5,136.3,134.2,130.4$, $129.3,128.9,128.9,128.7,128.6,128.0,127.1,127.0,126.2$, $124.1,122.5,122.3,121.9,121.8,119.9,119.6,119.6,119.2$, 119.1, 118.2, 111.8, 111.2, 111.1, 110.6, 109.2, 47.6, 44.6, 38.8, 23.4 ppm. IR (film) $\nu_{\max } 3271,3057,2923,1613,1495,1464$, $1450,1423,1350,1279,1183,1024,907,729,699 \mathrm{~cm}^{-1}$. HRMS (ESI) $m / z$ calcd for $\mathrm{C}_{33} \mathrm{H}_{27} \mathrm{~N}_{3} \mathrm{NaO} 504.2046[\mathrm{M}+$ $\mathrm{Na}]^{+}$, found 504.2048.

( \pm )-aS-13-Benzyl-6-((methoxy-d3)methyl-6,7,8, 13-tetrahydro-5H-benzo[6,7]azocino[5,4-b]indol-5-one-8-d (3a). According to procedure $\mathrm{B}$, lactam $3 \mathrm{a}$ was obtained from spiroindoline 1a $(18.2 \mathrm{mg}, 0.05 \mathrm{mmol})$ as a pale yellow solid
$(16.6 \mathrm{mg}, 83 \%$ yield, eluent: petroleum ether/ethyl acetate $=$ 3/1). Mp 108-109 ${ }^{\circ} \mathrm{C} .{ }^{1} \mathrm{H}$ NMR (400 MHz, $\left.\mathrm{CDCl}_{3}\right) \delta 7.61-$ $7.56(\mathrm{~m}, 1 \mathrm{H}), 7.53(\mathrm{dd}, J=7.7,1.5 \mathrm{~Hz}, 1 \mathrm{H}), 7.45(\mathrm{td}, J=7.5$, $1.3 \mathrm{~Hz}, 1 \mathrm{H}), 7.36(\mathrm{td}, J=7.6,1.5 \mathrm{~Hz}, 1 \mathrm{H}), 7.26-7.12(\mathrm{~m}$, $7 \mathrm{H}), 7.01-6.93(\mathrm{~m}, 2 \mathrm{H}), 5.17(\mathrm{~d}, J=17.0 \mathrm{~Hz}, 1 \mathrm{H}), 5.10-4.96$ $(\mathrm{m}, 2 \mathrm{H}), 4.88(\mathrm{dd}, J=10.3,2.0 \mathrm{~Hz}, 1 \mathrm{H}), 3.94-3.81(\mathrm{~m}, 1 \mathrm{H})$, 3.62 (ddd, $J=14.9,3.7,2.3 \mathrm{~Hz}, 1 \mathrm{H}$ ), 3.53 (dd, $J=13.8,6.1$ $\mathrm{Hz}, 0.51 \mathrm{H}), 2.96-2.87(\mathrm{~m}, 0.54 \mathrm{H}) \mathrm{ppm} .{ }^{13} \mathrm{C}$ NMR $(100$ $\left.\mathrm{MHz}, \mathrm{CDCl}_{3}\right) \delta 172.4,138.0,137.6,137.5,134.1,130.6,129.3$, $129.2,129.0,128.7,128.6(\mathrm{q}, J=3.0 \mathrm{~Hz}), 128.2,127.2,126.1$, $122.7119 .9,118.4,110.6,109.4,75.5,47.6,44.8,24.1$ (q, $J=$ $18.2 \mathrm{~Hz}$ ) ppm. IR (film) $\nu_{\max } 1648,1463,1448,1350,1183$, $1089,1008,967,906,728,700 \mathrm{~cm}^{-1}$. HRMS (ESI) $\mathrm{m} / z$ calcd for $\mathrm{C}_{26} \mathrm{H}_{20} \mathrm{D}_{4} \mathrm{~N}_{2} \mathrm{NaO}_{2} 423.1981[\mathrm{M}+\mathrm{Na}]^{+}$, found 423.1983 .

( \pm )-aS-13-Benzyl-6-((methoxy-d3)methyl)-4-methyl6,7,8,13-tetrahydro-5H-benzo[6,7]azocino[5,4-b]indol-5one-8-d (3b). According to procedure $\mathrm{B}$, lactam $3 \mathbf{b}$ was obtained from spiroindoline $\mathbf{1 b}(18.9 \mathrm{mg}, 0.05 \mathrm{mmol})$ as a pale yellow solid (17.0 mg, 82\% yield, eluent: petroleum ether/ ethyl acetate $=3 / 1) . \mathrm{Mp} 171-173{ }^{\circ} \mathrm{C}$. ${ }^{1} \mathrm{H}$ NMR $(400 \mathrm{MHz}$, $\left.\mathrm{CDCl}_{3}\right) \delta 7.58-7.53(\mathrm{~m}, 1 \mathrm{H}), 7.29-7.17(\mathrm{~m}, 5 \mathrm{H}), 7.17-7.09$ $(\mathrm{m}, 3 \mathrm{H}), 7.06-6.97(\mathrm{~m}, 3 \mathrm{H}), 5.23(\mathrm{~d}, J=16.9 \mathrm{~Hz}, 1 \mathrm{H}), 5.16-$ $5.05(\mathrm{~m}, 2 \mathrm{H}), 4.79(\mathrm{~d}, J=10.4 \mathrm{~Hz}, 1 \mathrm{H}), 3.91-3.75(\mathrm{~m}, 1 \mathrm{H})$, $3.62(\mathrm{dd}, J=14.9,6.4 \mathrm{~Hz}, 1 \mathrm{H}), 3.52(\mathrm{dd}, J=13.8,6.4 \mathrm{~Hz}$, $0.88 \mathrm{H}), 2.86(\mathrm{dd}, J=16.1,4.3 \mathrm{~Hz}, 0.2 \mathrm{H}), 2.41$ (s, 3H) ppm. ${ }^{13} \mathrm{C}$ NMR $\left(100 \mathrm{MHz}, \mathrm{CDCl}_{3}\right) \delta 171.6,138.1,137.4,137.0$, $135.5,134.7,130.8,129.2,128.8,128.7,128.5,128.0,127.1$, 126.2, 122.5, 119.7, 118.2, 110.7, 109.0 (d, $J=6.1 \mathrm{~Hz}), 74.9$, 47.7, 44.3 (d, $J=5.1 \mathrm{~Hz}), 23.8(\mathrm{q}, J=19.2 \mathrm{~Hz}), 19.8 \mathrm{ppm}$. IR (film) $\nu_{\max } 1645,1451,1423,1346,1098,1025,907,728,698$ $\mathrm{cm}^{-1}$. HRMS (ESI) $\mathrm{m} / z$ calcd for $\mathrm{C}_{27} \mathrm{H}_{22} \mathrm{D}_{4} \mathrm{~N}_{2} \mathrm{NaO}_{2} 437.2138$ $[\mathrm{M}+\mathrm{Na}]^{+}$, found 437.2136 .

( \pm )-aS-13-Benzyl-4-chloro-6-((methoxy-d3)methyl)6,7,8,13-tetrahydro-5H-benzo[6,7]azocino[5,4-b]indol-5one-8-d (3c). According to procedure $\mathrm{B}$, lactam $3 \mathrm{c}$ was obtained from spiroindoline $1 \mathrm{c}(20.0 \mathrm{mg}, 0.05 \mathrm{mmol})$ as a pale yellow solid ( $15.6 \mathrm{mg}, 72 \%$ yield, eluent: petroleum ether/ ethyl acetate $=2 / 1) . \mathrm{Mp} 195-196{ }^{\circ} \mathrm{C}$. ${ }^{1} \mathrm{H}$ NMR $(400 \mathrm{MHz}$, $\left.\mathrm{CDCl}_{3}\right) \delta 7.57(\mathrm{dt}, J=7.3,1.4 \mathrm{~Hz}, 1 \mathrm{H}), 7.47(\mathrm{dd}, J=8.1,1.1$ $\mathrm{Hz}, 1 \mathrm{H}), 7.30-7.21(\mathrm{~m}, 4 \mathrm{H}), 7.21-7.11(\mathrm{~m}, 3 \mathrm{H}), 7.08(\mathrm{dd}, J$ $=7.7,1.1 \mathrm{~Hz}, 1 \mathrm{H}), 7.05-7.00(\mathrm{~m}, 2 \mathrm{H}), 5.24(\mathrm{~d}, J=17.0 \mathrm{~Hz}$, $1 \mathrm{H}), 5.19-5.07(\mathrm{~m}, 2 \mathrm{H}), 4.77(\mathrm{~d}, J=10.5 \mathrm{~Hz}, 1 \mathrm{H}), 3.80$ (ddd, $J=15.0,13.8,1.1 \mathrm{~Hz}, 1 \mathrm{H}), 3.69(\mathrm{dd}, J=15.1,6.5 \mathrm{~Hz}, 1 \mathrm{H})$, $3.54(\mathrm{dd}, J=13.8,6.5 \mathrm{~Hz}, 0.82 \mathrm{H}), 2.88(\mathrm{ddd}, J=16.6,4.7,1.5$ $\mathrm{Hz}, 0.19 \mathrm{H})$ ppm. ${ }^{13} \mathrm{C}$ NMR $\left(100 \mathrm{MHz}, \mathrm{CDCl}_{3}\right) \delta 168.8$, $137.8,137.6,136.5,133.2,131.4,131.3,130.0,129.5,128.9$, $128.8,128.7,127.3,126.1,123.0,120.0,118.4,110.7,109.9$ (d, $J=5.1 \mathrm{~Hz}), 74.7,47.8,44.1(\mathrm{~d}, J=5.1 \mathrm{~Hz}), 23.8(\mathrm{q}, J=19.2$ $\mathrm{Hz}) \mathrm{ppm}$. IR (film) $\nu_{\max } 1655,1440,1419,1345,1229,1099$, $1018,910,729,699 \mathrm{~cm}^{-1}$. HRMS (ESI) $\mathrm{m} / z$ calcd for $\mathrm{C}_{26} \mathrm{H}_{19} \mathrm{D}_{4} \mathrm{ClN}_{2} \mathrm{NaO}_{2} 457.1591[\mathrm{M}+\mathrm{Na}]^{+}$, found 457.1590.

( \pm )-aS-13-Benzyl-6-((methoxy-d3)methyl)-3-methyl$6,7,8,13$-tetrahydro-5H-benzo[6,7]azocino[5,4-b]indol-5one-8-d (3d). According to procedure $\mathrm{B}$, lactam $3 \mathrm{~d}$ was obtained from spiroindoline $\mathbf{1 d}(18.9 \mathrm{mg}, 0.05 \mathrm{mmol})$ as a pale yellow solid (18.6 mg, 90\% yield, eluent: petroleum ether/ ethyl acetate $=3 / 1) . \mathrm{Mp} \mathrm{88-90}{ }^{\circ} \mathrm{C} .{ }^{1} \mathrm{H}$ NMR $(400 \mathrm{MHz}$, $\left.\mathrm{CDCl}_{3}\right) \delta 7.60-7.53(\mathrm{~m}, 1 \mathrm{H}), 7.35(\mathrm{~d}, J=1.8 \mathrm{~Hz}, 1 \mathrm{H}), 7.27-$ $7.10(\mathrm{~m}, 7 \mathrm{H}), 7.07(\mathrm{~d}, J=7.9 \mathrm{~Hz}, 1 \mathrm{H}), 7.00-6.94(\mathrm{~m}, 2 \mathrm{H})$, $5.17(\mathrm{~d}, J=16.9 \mathrm{~Hz}, 1 \mathrm{H}), 5.12-4.99(\mathrm{~m}, 2 \mathrm{H}), 4.86(\mathrm{dd}, J=$ 10.3, $2.0 \mathrm{~Hz}, 1 \mathrm{H}$ ), 3.90 (ddd, $J=14.4,9.1,4.8 \mathrm{~Hz}, 1 \mathrm{H}$ ), 3.62 (ddd, $J=14.9,3.7,2.3 \mathrm{~Hz}, 1 \mathrm{H}), 3.55-3.46(\mathrm{~m}, 0.52 \mathrm{H}), 2.92$ $(\mathrm{dd}, J=19.0,4.0 \mathrm{~Hz}, 0.61 \mathrm{H}), 2.40(\mathrm{~s}, 3 \mathrm{H}) \mathrm{ppm} .{ }^{13} \mathrm{C} \mathrm{NMR}$ 
$\left(100 \mathrm{MHz}, \mathrm{CDCl}_{3}\right) \delta 172.6,148.9,139.1,138.1,137.5,137.3$, $130.5,130.1,129.9$ (d, $J=6.1 \mathrm{~Hz}), 128.7,128.7,128.6,127.1$, $126.7,126.2,126.1(\mathrm{~d}, J=2.0 \mathrm{~Hz}), 125.2,122.5,119.8,118.3$, 110.6, $109.1(\mathrm{t}, J=3.0 \mathrm{~Hz}), 76.7,75.5(\mathrm{~d}, J=2.0 \mathrm{~Hz}), 47.5$, $44.8(\mathrm{t}, J=5.1 \mathrm{~Hz}), 24.1(\mathrm{q}, J=18.2 \mathrm{~Hz}), 21.2 \mathrm{ppm}$. IR (film) $\nu_{\max } 1644,1464,1437,1348,1307,1097,1022,908,830,730$, $698 \mathrm{~cm}^{-1}$. HRMS (ESI) $\mathrm{m} / z$ calcd for $\mathrm{C}_{27} \mathrm{H}_{22} \mathrm{D}_{4} \mathrm{~N}_{2} \mathrm{NaO}_{2}$ 437.2138 $[\mathrm{M}+\mathrm{Na}]^{+}$, found 437.2137.

( \pm )-aS-13-Benzyl-3-chloro-6-((methoxy-d3)methyl)6,7,8,13-tetrahydro-5H-benzo[6,7]azocino[5,4-b]indol-5one-8-d (3e). According to procedure $\mathrm{B}$, lactam $3 \mathrm{e}$ was obtained from spiroindoline $1 \mathrm{e}(20.0 \mathrm{mg}, 0.05 \mathrm{mmol})$ as a pale yellow solid (18.5 mg, $85 \%$ yield, eluent: petroleum ether/ ethyl acetate $=3 / 1)$. Mp $72-73{ }^{\circ} \mathrm{C} .{ }^{1} \mathrm{H}$ NMR $(400 \mathrm{MHz}$, $\left.\mathrm{CDCl}_{3}\right) \delta 7.58(\mathrm{dt}, J=6.7,1.6 \mathrm{~Hz}, 1 \mathrm{H}), 7.52(\mathrm{~d}, J=2.2 \mathrm{~Hz}$, $1 \mathrm{H}), 7.32(\mathrm{dd}, J=8.3,2.3 \mathrm{~Hz}, 1 \mathrm{H}), 7.30-7.06(\mathrm{~m}, 7 \mathrm{H}), 7.04-$ $6.88(\mathrm{~m}, 2 \mathrm{H}), 5.17(\mathrm{~d}, J=17.0 \mathrm{~Hz}, 1 \mathrm{H}), 5.10-4.95(\mathrm{~m}, 2 \mathrm{H})$, 4.87 (dd, $J=10.3,1.9 \mathrm{~Hz}, 1 \mathrm{H}), 3.86$ (ddd, $J=14.5$, 9.2, 4.8 $\mathrm{Hz}, 1 \mathrm{H}), 3.64$ (ddd, $J=15.0,3.8,2.3 \mathrm{~Hz}, 1 \mathrm{H}), 3.59-3.48$ (m, $0.47 \mathrm{H}$ ), 2.92 (ddd, $J=15.3,4.4,1.5 \mathrm{~Hz}, 0.53 \mathrm{H}) \mathrm{ppm} .{ }^{13} \mathrm{C}$ NMR (100 MHz, $\left.\mathrm{CDCl}_{3}\right) \delta 170.8,138.9,137.8,135.1,132.8$, $132.8,131.9,129.4,128.8,128.5$ (d, $J=3.0 \mathrm{~Hz}), 128.3,127.7$, $127.3,126.0,123.0,120.0,118.5,110.6,109.9,109.9(\mathrm{t}, J=3.0$ $\mathrm{Hz}), 75.6(\mathrm{~d}, J=2.0 \mathrm{~Hz}), 47.6,44.8(\mathrm{t}, J=5.1 \mathrm{~Hz}), 24.2(\mathrm{q}, J=$ $19.2 \mathrm{~Hz}$ ) ppm. IR (film) $\nu_{\max } 1648,1463,1437,1348,1223$, $1098,1020,908,832,730,697 \mathrm{~cm}^{-1}$. HRMS (ESI) $\mathrm{m} / z$ calcd for $\mathrm{C}_{26} \mathrm{H}_{19} \mathrm{D}_{4} \mathrm{ClN}_{2} \mathrm{NaO}_{2} 457.1591[\mathrm{M}+\mathrm{Na}]^{+}$, found 457.1591.

( \pm )-aS-13-Benzyl-3-fluoro-6-((methoxy-d3)methyl)6,7,8,13-tetrahydro-5H-benzo[6,7]azocino[5,4-b]indol-5one-8-d (3f). According to procedure B, lactam $3 \mathrm{f}$ was obtained from spiroindoline $1 \mathrm{f}(19.7 \mathrm{mg}, 0.05 \mathrm{mmol})$ as a pale yellow solid (16.3 mg, 78\% yield, eluent: petroleum ether/ ethyl acetate $=3 / 1)$. Mp 87-89 ${ }^{\circ} \mathrm{C} .{ }^{1} \mathrm{H}$ NMR $(400 \mathrm{MHz}$, $\left.\mathrm{CDCl}_{3}\right) \delta 7.64-7.49(\mathrm{~m}, 1 \mathrm{H}), 7.30-7.10(\mathrm{~m}, 8 \mathrm{H}), 7.06(\mathrm{td}, J$ $=8.3,2.7 \mathrm{~Hz}, 1 \mathrm{H}), 7.00-6.89(\mathrm{~m}, 2 \mathrm{H}), 5.17(\mathrm{~d}, J=17.0 \mathrm{~Hz}$, $1 \mathrm{H}), 5.09-4.95(\mathrm{~m}, 2 \mathrm{H}), 4.87$ (dd, $J=10.3,1.9 \mathrm{~Hz}, 1 \mathrm{H}), 3.86$ (ddd, $J=14.4,9.2,4.7 \mathrm{~Hz}, 1 \mathrm{H}), 3.65$ (ddd, $J=15.0,3.8,2.3$ $\mathrm{Hz}, 1 \mathrm{H}), 3.59-3.49(\mathrm{~m}, 0.51 \mathrm{H}), 2.92(\mathrm{dd}, J=15.5,3.7 \mathrm{~Hz}$, $0.55 \mathrm{H})$ ppm. ${ }^{13} \mathrm{C}$ NMR $\left(100 \mathrm{MHz}, \mathrm{CDCl}_{3}\right) \delta 170.9,164.0$, 161.5, 139.5 (d, $J=8.1 \mathrm{~Hz}), 137.7$ (d, $J=20.2 \mathrm{~Hz}$ ), 133.0, $132.6(\mathrm{~d}, J=8.1 \mathrm{~Hz}), 128.8,128.4(\mathrm{~d}, J=3.0 \mathrm{~Hz}), 127.3$, $126.0,125.3(\mathrm{~d}, J=3.0 \mathrm{~Hz}), 122.8,120.0,118.4,116.5(\mathrm{~d}, J=$ $21.2 \mathrm{~Hz}), 115.4$ (d, $J=23.2 \mathrm{~Hz}), 110.6,109.5,75.6,47.6,44.9$, $24.1(\mathrm{q}, J=19.2 \mathrm{~Hz}) \mathrm{ppm} .{ }^{19} \mathrm{~F} \operatorname{NMR}\left(376 \mathrm{MHz}, \mathrm{CDCl}_{3}\right) \delta$ $-111.48(\mathrm{~s}, 1 \mathrm{~F}) \mathrm{ppm}$. IR (film) $\nu_{\max } 1648,1464,1439,1346$, 1298, 1270, 1202, 197, 1021, 908, 833, 730, $698 \mathrm{~cm}^{-1}$. HRMS (ESI) $m / z$ calcd for $\mathrm{C}_{26} \mathrm{H}_{19} \mathrm{D}_{4} \mathrm{FN}_{2} \mathrm{NaO}_{2} 441.1887[\mathrm{M}+\mathrm{Na}]^{+}$, found 441.1885 .

( \pm )-aS-13-Benzyl-3-methoxy-6-((methoxy-d3)methyl)6,7,8,13-tetrahydro-5H-benzo[6,7]azocino[5,4-b]indol-5one-8-d (3g). According to procedure $\mathrm{B}$, lactam $3 \mathrm{~g}$ was obtained from spiroindoline $1 \mathrm{~g}(19.7 \mathrm{mg}, 0.05 \mathrm{mmol})$ as a pale yellow solid ( $19.4 \mathrm{mg}, 90 \%$ yield, eluent: petroleum ether/ ethyl acetate $=3 / 2)$. Mp 80-81 ${ }^{\circ} \mathrm{C} .{ }^{1} \mathrm{H}$ NMR $(400 \mathrm{MHz}$, $\left.\mathrm{CDCl}_{3}\right) \delta 7.56(\mathrm{dd}, J=8.2,1.8 \mathrm{~Hz}, 1 \mathrm{H}), 7.27-7.18(\mathrm{~m}, 3 \mathrm{H})$, 7.16-7.11 (m, 3H), 7.10-7.03 (m, 2H), 7.01-6.94 (m, 2H), $6.89(\mathrm{dd}, J=8.6,2.7 \mathrm{~Hz}, 1 \mathrm{H}), 5.17(\mathrm{~d}, J=16.9 \mathrm{~Hz}, 1 \mathrm{H}), 5.11-$ $4.97(\mathrm{~m}, 2 \mathrm{H}), 4.86(\mathrm{dd}, J=10.2,1.9 \mathrm{~Hz}, 1 \mathrm{H}), 3.90(\mathrm{ddd}, J=$ 14.5, 9.2, $4.8 \mathrm{~Hz}, 1 \mathrm{H}), 3.85$ (s, $3 \mathrm{H}), 3.63$ (ddd, $J=15.0,3.8$, $2.4 \mathrm{~Hz}, 1 \mathrm{H}), 3.56-3.45(\mathrm{~m}, 0.46 \mathrm{H}), 2.96-2.85(\mathrm{~m}, 0.54 \mathrm{H})$ ppm. ${ }^{13} \mathrm{C} \mathrm{NMR}\left(100 \mathrm{MHz}, \mathrm{CDCl}_{3}\right) \delta 172.1,160.0,138.7$, 138.1, 137.5, 134.0, 131.9, 128.7, 128.6 (d, $J=3.0 \mathrm{~Hz}), 127.1$, 126.1, 122.4, 121.4, 119.7, 118.2, 115.8 (d, $J=3.0 \mathrm{~Hz}$ ), 112.7, $110.5,108.8(\mathrm{t}, J=3.0 \mathrm{~Hz}), 75.6,55.5,47.5,44.9(\mathrm{~d}, J=5.1$ $\mathrm{Hz}$ ), 24.1 (q, $J=18.2 \mathrm{~Hz}$ ) ppm. IR (film) $\nu_{\max } 1644,1464$, $1452,1431,1349,1315,1293,1229,1182,1083,1032,1014$, $908,727,699 \mathrm{~cm}^{-1}$. HRMS (ESI) $\mathrm{m} / z$ calcd for $\mathrm{C}_{27} \mathrm{H}_{22} \mathrm{D}_{4} \mathrm{~N}_{2} \mathrm{NaO}_{3} 453.2087[\mathrm{M}+\mathrm{Na}]^{+}$, found 453.2085 .

( \pm )-aS-13-Benzyl-2,3-dimethoxy-6-((methoxy-d3)methyl)6,7,8, 13-tetrahydro-5H-benzo[6,7]azocino[5,4-b]indol-5one-8-d (3h). According to procedure $\mathrm{B}$, lactam $3 \mathrm{~h}$ was obtained from spiroindoline $\mathbf{1 h}(21.2 \mathrm{mg}, 0.05 \mathrm{mmol})$ as a pale yellow solid (18.4 mg, 83\% yield, eluent: petroleum ether/ ethyl acetate $=1 / 1) . \mathrm{Mp} 101-103{ }^{\circ} \mathrm{C} .{ }^{1} \mathrm{H}$ NMR $(400 \mathrm{MHz}$, $\left.\mathrm{CDCl}_{3}\right) \delta 7.60(\mathrm{dt}, J=6.9,1.5 \mathrm{~Hz}, 1 \mathrm{H}), 7.34-7.25(\mathrm{~m}, 3 \mathrm{H})$, $7.25-7.14(\mathrm{~m}, 4 \mathrm{H}), 7.12-6.97(\mathrm{~m}, 3 \mathrm{H}), 6.53(\mathrm{~s}, 1 \mathrm{H}), 5.21(\mathrm{~d}$, $J=17.5 \mathrm{~Hz}, 1 \mathrm{H}), 5.04(\mathrm{ddd} J=10.1,2.7,1.1 \mathrm{~Hz}, 1 \mathrm{H}), 4.96$ $(\mathrm{d}, J=17.5 \mathrm{~Hz}, 1 \mathrm{H}), 4.82(\mathrm{dd}, J=10.1,2.0 \mathrm{~Hz}, 1 \mathrm{H}), 4.00-$ 3.93 (m, 4H), 3.64 (ddd, $J=14.9,3.7,2.3 \mathrm{~Hz}, 1 \mathrm{H}), 3.55-3.46$ $(\mathrm{m}, 0.52 \mathrm{H}), 2.98-2.91(\mathrm{~m}, 0.59 \mathrm{H}), 3.18(\mathrm{~s}, 3 \mathrm{H}) \mathrm{ppm} .{ }^{13} \mathrm{C}$ NMR $\left(100 \mathrm{MHz}, \mathrm{CDCl}_{3}\right) \delta 172.3,149.3,149.2$, 138.8, 137.9, $134.2,134.2,129.2,128.9,128.4(\mathrm{~d}, J=3.0 \mathrm{~Hz}), 127.1,125.8$, $122.7,121.8,119.9,118.4,113.1,110.9,110.1,108.9(\mathrm{t}, J=3.0$ $\mathrm{Hz}$ ), 75.6, 56.1, 55.1, 47.7, 44.9 (d, $J=6.1 \mathrm{~Hz}), 24.0$ (q, $J=$ $16.2 \mathrm{~Hz}$ ) ppm. IR (film) $\nu_{\max } 1640,1604,1515,1465,1432$, $1262,1247,1206,1183,1096,1025,911,729,698 \mathrm{~cm}^{-1}$. HRMS (ESI) $m / z$ calcd for $\mathrm{C}_{28} \mathrm{H}_{24} \mathrm{D}_{4} \mathrm{~N}_{2} \mathrm{NaO}_{4} 483.2192[\mathrm{M}+$ $\mathrm{Na}]^{+}$, found 483.2191 .

( \pm )-aS-13-Benzyl-6-((methoxy-d3)methyl)-2-methyl6,7,8,13-tetrahydro-5H-benzo[6,7]azocino[5,4-b]indol-5one-8-d (3i). According to procedure B, lactam 3i was obtained from spiroindoline $\mathbf{1 i}(18.9 \mathrm{mg}, 0.05 \mathrm{mmol})$ as a pale yellow solid ( $17.6 \mathrm{mg}$, $85 \%$ yield, eluent: petroleum ether/ ethyl acetate $=3 / 1) . \mathrm{Mp} 68-70{ }^{\circ} \mathrm{C} .{ }^{1} \mathrm{H}$ NMR $(400 \mathrm{MHz}$, $\left.\mathrm{CDCl}_{3}\right) \delta 7.58(\mathrm{dd}, J=7.3,1.4 \mathrm{~Hz}, 1 \mathrm{H}), 7.43(\mathrm{~d}, J=7.8 \mathrm{~Hz}$, $1 \mathrm{H}), 7.29-7.11(\mathrm{~m}, 7 \mathrm{H}), 7.04-6.94(\mathrm{~m}, 2 \mathrm{H}), 6.92-6.85(\mathrm{~m}$, $1 \mathrm{H}), 5.20(\mathrm{~d}, J=16.9 \mathrm{~Hz}, 1 \mathrm{H}), 5.07-4.95(\mathrm{~m}, 2 \mathrm{H}), 4.86(\mathrm{dd}, J$ $=10.2,2.0 \mathrm{~Hz}, 1 \mathrm{H}), 3.97-3.82(\mathrm{~m}, 1 \mathrm{H}), 3.61$ (ddd, $J=14.9$, 3.7, $2.3 \mathrm{~Hz}, 1 \mathrm{H}), 3.55-3.47(\mathrm{~m}, 0.52 \mathrm{H}), 2.90(\mathrm{dd}, J=15.8,3.8$ $\mathrm{Hz}, 0.56 \mathrm{H}), 2.19$ (s, 3H) ppm. ${ }^{13} \mathrm{C} \mathrm{NMR}\left(100 \mathrm{MHz}^{\mathrm{C}} \mathrm{CDCl}_{3}\right)$ $\delta 172.5,139.2,138.3,137.7,134.5,134.3,131.4,129.6,129.1$, $128.6,128.5$ (d, $J=3.0 \mathrm{~Hz}), 128.2,127.1,126.4,122.6,119.8$, 118.4, 110.4, 109.3 (d, $J=3.0 \mathrm{~Hz}), 75.6(\mathrm{~d}, J=2.0 \mathrm{~Hz}), 47.7$, $44.9(\mathrm{~d}, J=5.1 \mathrm{~Hz}), 24.1(\mathrm{q}, J=18.2 \mathrm{~Hz}), 21.1 \mathrm{ppm}$. IR (film) $\nu_{\max } 1642,1464,1452,1432,1348,1095,1023,908,829,728$, $699 \mathrm{~cm}^{-1}$. HRMS (ESI) $\mathrm{m} / z$ calcd for $\mathrm{C}_{27} \mathrm{H}_{22} \mathrm{D}_{4} \mathrm{~N}_{2} \mathrm{NaO}_{2}$ 437.2138 $[\mathrm{M}+\mathrm{Na}]^{+}$, found 437.2136.

( \pm )-aS-13-Benzyl-2-fluoro-6-((methoxy-d3)methyl)6,7,8,13-tetrahydro-5H-benzo[6,7]azocino[5,4-b]indol-5one-8-d (3j). According to procedure $B$, lactam $3 \mathbf{j}$ was obtained from spiroindoline $\mathbf{1 j}(19.1 \mathrm{mg}, 0.05 \mathrm{mmol})$ as a pale yellow solid (16.7 mg, $80 \%$ yield, eluent: petroleum ether/ ethyl acetate $=3 / 1) . \mathrm{Mp} 212-213{ }^{\circ} \mathrm{C} .{ }^{1} \mathrm{H}$ NMR $(400 \mathrm{MHz}$, $\left.\mathrm{CDCl}_{3}\right) \delta 7.59(\mathrm{dd}, J=7.6,1.3 \mathrm{~Hz}, 1 \mathrm{H}), 7.52(\mathrm{dd}, J=8.5,5.8$ $\mathrm{Hz}, 1 \mathrm{H}), 7.29-7.09(\mathrm{~m}, 7 \mathrm{H}), 6.98-6.91(\mathrm{~m}, 2 \mathrm{H}), 6.87$ (dd, J $=9.4,2.5 \mathrm{~Hz}, 1 \mathrm{H}), 5.20(\mathrm{~d}, J=16.9 \mathrm{~Hz}, 1 \mathrm{H}), 5.07(\mathrm{~d}, J=16.9$ $\mathrm{Hz}, 1 \mathrm{H}), 4.99$ (ddd, $J=10.3,2.7,1.0 \mathrm{~Hz}, 1 \mathrm{H}), 4.88$ (dd, $J=$ 10.3, $2.0 \mathrm{~Hz}, 1 \mathrm{H}$ ), 3.86 (ddd, $J=14.4,9.1,4.7 \mathrm{~Hz}, 1 \mathrm{H}$ ), 3.64 (ddd, $J=15.0,3.7,2.3 \mathrm{~Hz}, 1 \mathrm{H}), 3.59-3.50(\mathrm{~m}, 0.52 \mathrm{H}), 2.96-$ $2.89(\mathrm{~m}, 0.58 \mathrm{H}) \mathrm{ppm} .{ }^{13} \mathrm{C} \mathrm{NMR}\left(100 \mathrm{MHz}, \mathrm{CDCl}_{3}\right) \delta 171.5$, $163.9,161.4,137.7$ (d, $J=17.2 \mathrm{~Hz}), 133.6,132.7,131.7$ (d, $J=$ $8.1 \mathrm{~Hz}), 130.4(\mathrm{~d}, J=9.1 \mathrm{~Hz}), 128.8,128.4(\mathrm{~d}, J=3.0 \mathrm{~Hz})$, $127.3,126.0,123.1,120.1,118.6,117.5(\mathrm{~d}, J=22.2 \mathrm{~Hz}), 116.1$ (d, $J=22.2 \mathrm{~Hz}), 110.6,110.2(\mathrm{~d}, J=3.0 \mathrm{~Hz}), 75.7,47.7,44.9$, $24.2(\mathrm{q}, J=18.2 \mathrm{~Hz})$ ppm. ${ }^{19} \mathrm{~F} \operatorname{NMR}\left(376 \mathrm{MHz} \mathrm{CDCl}_{3}\right) \delta$ -110.52 (s, 1F) ppm. IR (film) $\nu_{\max } 1644,1605,1464,1453$, 
1434, 1345, 1294, 1262, 1232, 1189, 1099, 1022, 909, 728, 699 $\mathrm{cm}^{-1}$. HRMS (ESI) $\mathrm{m} / z$ calcd for $\mathrm{C}_{26} \mathrm{H}_{19} \mathrm{D}_{4} \mathrm{FN}_{2} \mathrm{NaO}_{2}$ $441.1887[\mathrm{M}+\mathrm{Na}]^{+}$, found 441.1887 .

( \pm )-aS-13-Benzyl-2-methoxy-6-((methoxy-d3)methyl)6,7,8,13-tetrahydro-5H-benzo[6,7]azocino[5,4-b]indol-5one-8-d (3k). According to procedure $\mathrm{B}$, lactam $3 \mathrm{k}$ was obtained from spiroindoline $1 \mathrm{k}(19.7 \mathrm{mg}, 0.05 \mathrm{mmol})$ as a pale yellow solid ( $18.9 \mathrm{mg}, 88 \%$ yield, eluent: petroleum ether/ ethyl acetate $=3 / 2)$. Mp 83-84 ${ }^{\circ} \mathrm{C} .{ }^{1} \mathrm{H}$ NMR $(400 \mathrm{MHz}$, $\left.\mathrm{CDCl}_{3}\right) \delta 7.60(\mathrm{dt}, J=7.1,1.4 \mathrm{~Hz}, 1 \mathrm{H}), 7.46(\mathrm{~d}, J=8.5 \mathrm{~Hz}$, $1 \mathrm{H}), 7.32-7.13(\mathrm{~m}, 6 \mathrm{H}), 7.07-6.99(\mathrm{~m}, 2 \mathrm{H}), 6.95(\mathrm{dd}, J=$ $8.5,2.6 \mathrm{~Hz}, 1 \mathrm{H}), 6.62(\mathrm{~d}, J=2.5 \mathrm{~Hz}, 1 \mathrm{H}), 5.21(\mathrm{~d}, J=17.2 \mathrm{~Hz}$, $1 \mathrm{H}), 5.09-4.96(\mathrm{~m}, 2 \mathrm{H}), 4.85$ (dd, $J=10.2,2.1 \mathrm{~Hz}, 1 \mathrm{H}), 3.92$ (ddd, $J=14.4,9.1,4.7 \mathrm{~Hz}, 1 \mathrm{H}), 3.62$ (ddd, $J=14.9,3.7,2.2$ $\mathrm{Hz}, 1 \mathrm{H}), 3.57-3.48(\mathrm{~m}, 0.52 \mathrm{H}), 2.97-2.88(\mathrm{~m}, 0.56 \mathrm{H})], 3.34$ (s, 3H) ppm. $\left.{ }^{13} \mathrm{C} \mathrm{NMR} \mathrm{(100} \mathrm{MHz}, \mathrm{CDCl}_{3}\right) \delta 172.4,159.9$, 138.5, 137.9, 134.1, 130.7, 129.9, 129.5 (d, $J=1.0 \mathrm{~Hz}), 128.8$, $128.4(\mathrm{~d}, J=3.0 \mathrm{~Hz}), 127.1,125.9,122.8,119.9,118.5$ (d, $J=$ $2.0 \mathrm{~Hz}), 116.0,114.5,110.4,109.5(\mathrm{t}, J=3.0 \mathrm{~Hz}), 75.6,54.8$, 47.8, $44.8(\mathrm{t}, J=5.1 \mathrm{~Hz}), 24.1(\mathrm{q}, J=19.2 \mathrm{~Hz}) \mathrm{ppm}$. IR (film) $\nu_{\max } 1642,1602,1465,1433,1308,1237,1214,1094,1021$, $909,730,700 \mathrm{~cm}^{-1}$. HRMS (ESI) $\mathrm{m} / z$ calcd for $\mathrm{C}_{27} \mathrm{H}_{22} \mathrm{D}_{4} \mathrm{~N}_{2} \mathrm{NaO}_{3} 453.2087[\mathrm{M}+\mathrm{Na}]^{+}$, found 453.2085 .

( \pm )-aS-13-Benzyl-6-((methoxy-d3)methyl)-10-methyl6,7,8,13-tetrahydro-5H-benzo[6,7]azocino[5,4-b]indol-5one-8-d (3I). According to procedure B, lactam 31 was obtained from spiroindoline 11 (18.9 $\mathrm{mg}, 0.05 \mathrm{mmol})$ as a pale yellow solid ( $18.4 \mathrm{mg}$, $89 \%$ yield, eluent: petroleum ether/ ethyl acetate $=3 / 1) . \mathrm{Mp} 77-78{ }^{\circ} \mathrm{C} .{ }^{1} \mathrm{H}$ NMR $(400 \mathrm{MHz}$, $\left.\mathrm{CDCl}_{3}\right) \delta 7.53(\mathrm{dd}, J=7.6,1.5 \mathrm{~Hz}, 1 \mathrm{H}), 7.43(\mathrm{td}, J=7.5,1.3$ $\mathrm{Hz}, 1 \mathrm{H}), 7.39-7.32(\mathrm{~m}, 2 \mathrm{H}), 7.27-7.15(\mathrm{~m}, 4 \mathrm{H}), 7.07-6.92$ $(\mathrm{m}, 4 \mathrm{H}), 5.14(\mathrm{~d}, J=16.9 \mathrm{~Hz}, 1 \mathrm{H}), 5.09-4.99(\mathrm{~m}, 2 \mathrm{H}), 4.86$ (dd, $J=10.3,1.9 \mathrm{~Hz}, 1 \mathrm{H}), 3.86$ (ddd, $J=14.4,9.2,4.8 \mathrm{~Hz}$, $1 \mathrm{H}), 3.62$ (ddd, $J=15.0,3.8,2.3 \mathrm{~Hz}, 1 \mathrm{H}), 3.54-3.45(\mathrm{~m}$, $0.51 \mathrm{H}), 2.94-2.85(\mathrm{~m}, 0.57 \mathrm{H}), 2.46(\mathrm{~s}, 3 \mathrm{H}) \mathrm{ppm} .{ }^{13} \mathrm{C} \mathrm{NMR}$ $\left(100 \mathrm{MHz}, \mathrm{CDCl}_{3}\right) \delta 138.1,137.5,136.0,134.1(\mathrm{~d}, J=2.0$ $\mathrm{Hz}), 130.5,129.4,129.2,129.1,128.9,128.8(\mathrm{~d}, J=3.0 \mathrm{~Hz})$, 128.6, 128.2, 127.1, 126.1, 124.2, 118.1, $108.9(\mathrm{t}, J=3.0 \mathrm{~Hz})$, $75.5(\mathrm{~d}, J=2.0 \mathrm{~Hz}), 47.6,44.8(\mathrm{~d}, J=6.1 \mathrm{~Hz}), 24.1(\mathrm{q}, J=$ $18.2 \mathrm{~Hz}$ ), $21.5 \mathrm{ppm}$. IR (film) $\nu_{\max } 1648,1466,1443,1421$, 1351, 1306, 1098, 1019, 909, 791, 773, 729, $699 \mathrm{~cm}^{-1}$. HRMS (ESI) $\mathrm{m} / z$ calcd for $\mathrm{C}_{27} \mathrm{H}_{22} \mathrm{D}_{4} \mathrm{~N}_{2} \mathrm{NaO}_{2}$ 437.2138 $[\mathrm{M}+\mathrm{Na}]^{+}$, found 437.2135.

( \pm )-aS-13-Benzyl-10-chloro-6-((methoxy-d3)methyl)6,7,8,13-tetrahydro-5H-benzo[6,7]azocino[5,4-b]indol-5one-8-d (3m). According to procedure $\mathrm{B}$, lactam $3 \mathrm{~m}$ was obtained from spiroindoline $1 \mathrm{~m}(20.0 \mathrm{mg}, 0.05 \mathrm{mmol})$ as a pale yellow solid ( $15.0 \mathrm{mg}$, 69\% yield, eluent: petroleum ether/ ethyl acetate $=2 / 1)$. Mp 73-74 ${ }^{\circ} \mathrm{C} .{ }^{1} \mathrm{H}$ NMR $(400 \mathrm{MHz}$, $\left.\mathrm{CDCl}_{3}\right) \delta 7.54(\mathrm{td}, J=3.6,2.8,1.5 \mathrm{~Hz}, 2 \mathrm{H}), 7.47(\mathrm{td}, J=7.5$, $1.3 \mathrm{~Hz}, 1 \mathrm{H}), 7.38(\mathrm{td}, J=7.6,1.6 \mathrm{~Hz}, 1 \mathrm{H}), 7.28-7.16(\mathrm{~m}$, $4 \mathrm{H}), 7.11(\mathrm{dd}, J=8.7,2.0 \mathrm{~Hz}, 1 \mathrm{H}), 7.05(\mathrm{~d}, J=8.7 \mathrm{~Hz}, 1 \mathrm{H})$, $6.93(\mathrm{dd}, J=7.8,1.7 \mathrm{~Hz}, 2 \mathrm{H}), 5.15(\mathrm{~d}, J=16.9 \mathrm{~Hz}, 1 \mathrm{H}), 5.09-$ $4.95(\mathrm{~m}, 2 \mathrm{H}), 4.91(\mathrm{dd}, J=10.3,2.0 \mathrm{~Hz}, 1 \mathrm{H}), 3.85(\mathrm{ddd}, J=$ 14.5, 9.2, $4.8 \mathrm{~Hz}, 1 \mathrm{H}), 3.61$ (ddd, $J=15.1,3.8,2.4 \mathrm{~Hz}, 1 \mathrm{H})$, $3.50-3.40(\mathrm{~m}, 0.52 \mathrm{H}), 2.88$ (ddd, $J=14.8,4.4,1.9 \mathrm{~Hz}, 0.57 \mathrm{H})$ ppm. ${ }^{13} \mathrm{C}$ NMR $\left(100 \mathrm{MHz}, \mathrm{CDCl}_{3}\right) \delta 172.2,137.5,137.5$, $135.9,135.4$ (d, $J=2.0 \mathrm{~Hz}), 129.6(\mathrm{~d}, J=3.0 \mathrm{~Hz}), 129.4$, $129.3,128.8,128.7,128.2,127.3,126.0,125.6,122.9,117.9$, 111.7, $109.1(\mathrm{t}, J=3.0 \mathrm{~Hz}), 75.7,47.7,44.9(\mathrm{~d}, J=5.1 \mathrm{~Hz})$, 24.2 (q, $J=18.2 \mathrm{~Hz}$ ) ppm. IR (film) $\nu_{\max } 1644,1465,1440$, $1421,1351,1032,1096,1018,908,788,765,728,699 \mathrm{~cm}^{-1}$.
HRMS (ESI) $m / z$ calcd for $\mathrm{C}_{26} \mathrm{H}_{19} \mathrm{D}_{4} \mathrm{ClN}_{2} \mathrm{NaO}_{2} 457.1591[\mathrm{M}$ $+\mathrm{Na}]^{+}$, found 457.1592 .

( \pm )-aS-13-Benzyl-10-methoxy-6-((methoxy-d3)methyl)6,7,8,13-tetrahydro-5H-benzo[6,7]azocino[5,4-b]indol-5one-8-d (3n). According to procedure B, lactam 3n was obtained from spiroindoline $1 \mathrm{n}(19.7 \mathrm{mg}, 0.05 \mathrm{mmol})$ as a pale yellow solid ( $18.1 \mathrm{mg}, 84 \%$ yield, eluent: petroleum ether/ ethyl acetate $=3 / 1) . \mathrm{Mp} 80-81{ }^{\circ} \mathrm{C} .{ }^{1} \mathrm{H}$ NMR $(400 \mathrm{MHz}$, $\left.\mathrm{CDCl}_{3}\right) \delta 7.46(\mathrm{dd}, J=7.6,1.5 \mathrm{~Hz}, 1 \mathrm{H}), 7.37(\mathrm{td}, J=7.5,1.3$ $\mathrm{Hz}, 1 \mathrm{H}), 7.29(\mathrm{td}, J=7.5,1.5 \mathrm{~Hz}, 1 \mathrm{H}), 7.20-7.09(\mathrm{~m}, 5 \mathrm{H})$, $7.00-6.92(\mathrm{~m}, 1 \mathrm{H}), 6.91-6.86(\mathrm{~m}, 2 \mathrm{H}), 6.75(\mathrm{~d}, J=8.9 \mathrm{~Hz}$, $1 \mathrm{H}), 5.06(\mathrm{~d}, J=16.9 \mathrm{~Hz}, 1 \mathrm{H}), 5.01-4.91(\mathrm{~m}, 2 \mathrm{H}), 4.84(\mathrm{dd}, J$ $=10.3,2.1 \mathrm{~Hz}, 1 \mathrm{H}), 3.84-3.77(\mathrm{~m}, 4 \mathrm{H}), 3.55(\mathrm{ddd}, J=15.0$, 3.8, $2.3 \mathrm{~Hz}, 1 \mathrm{H}), 3.43-3.35(\mathrm{~m}, 0.49 \mathrm{H}), 2.87-2.77(\mathrm{~m}$, $0.52 \mathrm{H})$ ppm. ${ }^{13} \mathrm{C} \mathrm{NMR}\left(100 \mathrm{MHz}, \mathrm{CDCl}_{3}\right) \delta 172.3,154.3$, $138.0,137.4,134.7,132.9,130.5,129.3,129.3,128.9,128.7$, $128.2,127.1,126.1,112.6,111.4,111.3,109.0,100.3,75.6$, 55.9, 47.7, 44.9, $24.3\left(\mathrm{q}, J=17.2 \mathrm{~Hz}\right.$ ) ppm. IR (film) $\nu_{\max }$ $1648,1477,1466,1433,1304,1209,1097,1053,1019,911$, 787, 772, 732, $700 \mathrm{~cm}^{-1}$. HRMS (ESI) $\mathrm{m} / z$ calcd for $\mathrm{C}_{27} \mathrm{H}_{22} \mathrm{D}_{4} \mathrm{~N}_{2} \mathrm{NaO}_{3} 453.2087[\mathrm{M}+\mathrm{Na}]^{+}$, found 453.2086 .

( \pm )-aS-6-((Methoxy-d3)methyl)-13-methyl-6,7,8, 13-tetrahydro-5H-benzo[6,7]azocino[5,4-b]indol-5-one-8-d (3o). According to procedure $\mathrm{B}$, lactam 30 was obtained from spiroindoline $10(14.4 \mathrm{mg}, 0.05 \mathrm{mmol})$ as a pale yellow solid $(14.8 \mathrm{mg}, 91 \%$ yield, eluent: petroleum ether $/$ ethyl acetate $=$ 2/1). Mp 61-63 ${ }^{\circ} \mathrm{C} .{ }^{1} \mathrm{H}$ NMR (400 MHz, $\left.\mathrm{CDCl}_{3}\right) \delta 7.61-$ $7.44(\mathrm{~m}, 4 \mathrm{H}), 7.36-7.22(\mathrm{~m}, 3 \mathrm{H}), 7.15$ (ddd, $J=7.9,6.7,1.2$ $\mathrm{Hz}, 1 \mathrm{H}), 5.06$ (ddd, $J=10.2,2.7,1.1 \mathrm{~Hz}, 1 \mathrm{H}), 4.78$ (dd, $J=$ $10.2,1.8 \mathrm{~Hz}, 1 \mathrm{H}), 3.93-3.79(\mathrm{~m}, 1 \mathrm{H}), 3.62(\mathrm{ddd}, J=15.0,3.7$, $2.2 \mathrm{~Hz}, 1 \mathrm{H}), 3.51(\mathrm{~s}, 3 \mathrm{H}), 3.50-3.46(\mathrm{~m}, 0.55 \mathrm{H}), 2.92-2.82$ $(\mathrm{m}, 0.53 \mathrm{H})$ ppm. ${ }^{13} \mathrm{C}$ NMR $\left(100 \mathrm{MHz}, \mathrm{CDCl}_{3}\right) \delta$ 172.4, 138.0, 137.2, 133.9 (d, $J=2.0 \mathrm{~Hz}$ ), 131.0, 129.4, 129.3, 128.8, $128.2,128.1$ (d, $J=3.0 \mathrm{~Hz}), 122.5,119.5,118.3,109.5,108.8$ $(\mathrm{t}, J=3.0 \mathrm{~Hz}), 75.3,44.5,31.0,24.4(\mathrm{q}, J=19.2 \mathrm{~Hz}) \mathrm{ppm}$. IR (film) $\nu_{\max } 1643,1467,1444,1422,1358,1232,1097,1031$, 1012, 910, 734, $645 \mathrm{~cm}^{-1}$. HRMS (ESI) $\mathrm{m} / z$ calcd for $\mathrm{C}_{20} \mathrm{H}_{16} \mathrm{D}_{4} \mathrm{~N}_{2} \mathrm{NaO}_{2} 347.1668[\mathrm{M}+\mathrm{Na}]^{+}$, found 347.1667.

14-Benzyl-6-(methoxymethyl)-7,8,9,14-tetrahydrobenzo[3,4]azonino[5,6-b]indol-5(6H)-one (5a). According to procedure A, lactam 5a was obtained from spiroindoline 4 (18.9 $\mathrm{mg}, 0.05 \mathrm{mmol})$ and methanol $(1.65 \mathrm{mg}, 0.05 \mathrm{mmol})$ as a pale yellow solid (18.1 mg, $88 \%$ yield, eluent: petroleum ether/ ethyl acetate $=3 / 1) . \mathrm{Mp} 47-49{ }^{\circ} \mathrm{C} .{ }^{1} \mathrm{H}$ NMR $(400 \mathrm{MHz}$, $\left.\mathrm{CDCl}_{3}\right) \delta 7.62-7.56(\mathrm{~m}, 1 \mathrm{H}), 7.48(\mathrm{td}, J=7.6,1.2 \mathrm{~Hz}, 1 \mathrm{H})$, $7.39(\mathrm{dd}, J=7.5,1.4 \mathrm{~Hz}, 1 \mathrm{H}), 7.29(\mathrm{td}, J=7.6,1.4 \mathrm{~Hz}, 1 \mathrm{H})$, 7.24-7.17 (m, 3H), 7.16-7.07 (m, 4H), 7.00-6.91 (m, 2H), $5.23(\mathrm{~d}, J=16.9 \mathrm{~Hz}, 1 \mathrm{H}), 4.86(\mathrm{~d}, J=16.9 \mathrm{~Hz}, 1 \mathrm{H}), 4.72(\mathrm{dd}$, $J=10.4,1.0 \mathrm{~Hz}, 1 \mathrm{H}), 4.57(\mathrm{~d}, J=10.4 \mathrm{~Hz}, 1 \mathrm{H}), 3.53-3.30$ (m, $2 \mathrm{H}$ ), 3.12 (dddd, $J=14.5,5.2,2.3,1.0 \mathrm{~Hz}, 1 \mathrm{H}), 2.81$ (s, $3 \mathrm{H}$ ), 2.36 (ddd, $J=14.5,13.0,2.5 \mathrm{~Hz}, 1 \mathrm{H}$ ), 2.02 (dddt, $J=$ 15.2, 13.2, 11.0, $2.2 \mathrm{~Hz}, 1 \mathrm{H}), 1.73-1.67(\mathrm{~m}, 1 \mathrm{H}) \mathrm{ppm} .{ }^{13} \mathrm{C}$ NMR $\left(100 \mathrm{MHz}, \mathrm{CDCl}_{3}\right) \delta 171.7,140.7,138.6,136.7,134.7$, $130.9,129.52,129.0,128.5,128.2,127.1,127.0,126.3,124.8$, 122.0, 119.4, 118.1, 114.5, 110.2, 75.5, 55.8, 49.8, 47.5, 27.2, 24.5 ppm. IR (film) $\nu_{\max } 2923,1640,1460,1442,1421,1388$, 1367, 1351, 1329, 1178, 1094, 1063, 1030, 762, 731, $696 \mathrm{~cm}^{-1}$. HRMS (ESI) $m / z$ calcd for $\mathrm{C}_{27} \mathrm{H}_{26} \mathrm{~N}_{2} \mathrm{NaO}_{2} 433.1886[\mathrm{M}+$ $\mathrm{Na}]^{+}$, found 433.1886 .

14-Benzyl-6-((2-hydroxynaphthalen-1-yl)methyl)7,8,9,14-tetrahydrobenzo[3,4]azonino[5,6-b]indol-5(6H)one $(\mathbf{5 b})$. According to procedure A, lactam $\mathbf{5 b}$ was obtained from spiroindoline $4(18.9 \mathrm{mg}, 0.05 \mathrm{mmol})$ and 2 - 
hydroxynaphthalene $(7.2 \mathrm{mg}, 0.05 \mathrm{mmol})$ as a white solid (23.6 mg, 91\% yield, eluent: petroleum ether $/$ ethyl acetate $=$ 1/1). Mp 258-260 ${ }^{\circ} \mathrm{C} .{ }^{1} \mathrm{H}$ NMR $\left(400 \mathrm{MHz}, \mathrm{CDCl}_{3}\right) \delta 9.93$ $(\mathrm{s}, 1 \mathrm{H}), 7.82(\mathrm{~d}, J=8.6 \mathrm{~Hz}, 1 \mathrm{H}), 7.74(\mathrm{~d}, J=8.1 \mathrm{~Hz}, 1 \mathrm{H})$, $7.65(\mathrm{~d}, J=8.9 \mathrm{~Hz}, 1 \mathrm{H}), 7.53(\mathrm{~d}, J=7.7 \mathrm{~Hz}, 1 \mathrm{H}), 7.45$ (q, $J=$ $7.6 \mathrm{~Hz}, 2 \mathrm{H}), 7.36(\mathrm{~d}, J=7.5 \mathrm{~Hz}, 1 \mathrm{H}), 7.34-7.07(\mathrm{~m}, 9 \mathrm{H})$, $7.07-6.90(\mathrm{~m}, 3 \mathrm{H}), 5.30(\mathrm{~d}, J=16.9 \mathrm{~Hz}, 1 \mathrm{H}), 5.01-4.75(\mathrm{~m}$, $2 \mathrm{H}), 4.55(\mathrm{~d}, J=15.5 \mathrm{~Hz}, 1 \mathrm{H}), 3.54-3.29(\mathrm{~m}, 2 \mathrm{H}), 3.15$ (dd, $J$ $=13.9,4.6 \mathrm{~Hz}, 1 \mathrm{H}), 2.45-2.30(\mathrm{~m}, 1 \mathrm{H}), 2.20(\mathrm{q}, J=12.8 \mathrm{~Hz}$, $1 \mathrm{H}), 1.74(\mathrm{t}, J=10.0 \mathrm{~Hz}, 1 \mathrm{H}) \mathrm{ppm} .{ }^{13} \mathrm{C} \mathrm{NMR}(100 \mathrm{MHz}$, $\left.\mathrm{CDCl}_{3}\right) \delta 172.7,155.6,139.2,138.5,136.8,134.4,134.0$, $130.9,130.5,129.6,129.3,129.0,128.7,128.6,128.5,127.1$, $126.9,126.7,126.2,125.4,122.5,122.2,121.0,119.9,119.6$, 118.0, 114.0, 112.7, 110.4, 50.3, 47.6, 38.1, 25.8, 24.3 ppm. IR (film) $\nu_{\max } 3141,1590,1579,1507,1497,1461,1435,1409$, $1368,1347,1272,1251,817,743,729 \mathrm{~cm}^{-1}$. HRMS (ESI) m/ $z$ calcd for $\mathrm{C}_{36} \mathrm{H}_{30} \mathrm{~N}_{2} \mathrm{NaO}_{2}$ 545.2199 $[\mathrm{M}+\mathrm{Na}]^{+}$, found 545.2200

6-((1H-Indol-3-yl)methyl)-14-benzyl-7,8,9,14tetrahydrobenzo[3,4]azonino[5,6-b]indol-5(6H)-one (5c). According to procedure A, lactam 5c was obtained from spiroindoline 4 (18.9 $\mathrm{mg}, 0.05 \mathrm{mmol})$ and indole $(5.9 \mathrm{mg}, 0.05$ $\mathrm{mmol})$ as a white solid $(21.0 \mathrm{mg}, 85 \%$ yield, eluent: petroleum ether/ethyl acetate $=1 / 1) . \mathrm{Mp} 164-166{ }^{\circ} \mathrm{C} .{ }^{1} \mathrm{H}$ NMR $(400$ $\left.\mathrm{MHz} \mathrm{CDCl}_{3}\right) \delta 8.05(\mathrm{~d}, J=7.7 \mathrm{~Hz}, 1 \mathrm{H}), 7.86(\mathrm{~s}, 1 \mathrm{H}), 7.34-$ $7.19(\mathrm{~m}, 5 \mathrm{H}), 7.19-7.02(\mathrm{~m}, 4 \mathrm{H}), 6.92(\mathrm{td}, J=7.5,4.9 \mathrm{~Hz}$, $2 \mathrm{H}), 6.65(\mathrm{q}, J=8.0 \mathrm{~Hz}, 2 \mathrm{H}), 6.52(\mathrm{~d}, J=7.8 \mathrm{~Hz}, 1 \mathrm{H}), 6.34$ $(\mathrm{d}, J=8.3 \mathrm{~Hz}, 1 \mathrm{H}), 6.28(\mathrm{~d}, J=7.8 \mathrm{~Hz}, 1 \mathrm{H}), 6.15(\mathrm{~d}, J=2.7$ $\mathrm{Hz}, 1 \mathrm{H}), 4.58(\mathrm{td}, J=12.3,7.1 \mathrm{~Hz}, 1 \mathrm{H}), 4.21(\mathrm{~d}, J=16.2 \mathrm{~Hz}$, $1 \mathrm{H}), 4.03(\mathrm{~d}, J=16.2 \mathrm{~Hz}, 1 \mathrm{H}), 3.89$ (d, $J=13.3 \mathrm{~Hz}, 1 \mathrm{H}), 3.47$ (d, $J=13.3 \mathrm{~Hz}, 1 \mathrm{H}), 3.15$ (dd, $J=13.1,7.2 \mathrm{~Hz}, 1 \mathrm{H}), 2.64$ (d, $J$ $=13.2 \mathrm{~Hz}, 1 \mathrm{H}), 2.49(\mathrm{dd}, J=15.6,7.1 \mathrm{~Hz}, 1 \mathrm{H}), 2.35-2.15(\mathrm{~m}$, $1 \mathrm{H}), 2.07-1.89(\mathrm{~m}, 1 \mathrm{H}) \mathrm{ppm} .{ }^{13} \mathrm{C} \mathrm{NMR}\left(100 \mathrm{MHz}, \mathrm{CDCl}_{3}\right)$ $\delta 167.4,150.7,140.9,138.3,136.0,133.1,131.0,128.7,128.6$, $128.4,127.9,126.7,126.3,126.1,125.9,124.3,123.7,121.0$, $120.5,120.2,119.4,119.3,110.5,110.2,58.6,48.7,46.8,46.5$, $22.6 \mathrm{ppm}$. IR (film) $\nu_{\max } 3306,2928,1641,1602,1481,1459$, 1421, 1348, 1296, 1236, 731, 707, $697 \mathrm{~cm}^{-1}$. HRMS (ESI) $\mathrm{m} /$ $z$ calcd for $\mathrm{C}_{34} \mathrm{H}_{29} \mathrm{~N}_{3} \mathrm{NaO} 518.2203[\mathrm{M}+\mathrm{Na}]^{+}$, found 518.2205 .

( \pm )-aS-14-Benzyl-6-((methoxy-d3)methyl)-7,8,9, 14tetrahydrobenzo[3,4]azonino[5,6-b]indol-5(6H)-one-9-d $(5 d)$. According to procedure B, lactam $5 \mathrm{~d}$ was obtained from spiroindoline $4(18.9 \mathrm{mg}, 0.05 \mathrm{mmol})$ as a pale yellow solid $(18.6 \mathrm{mg}, 90 \%$ yield, eluent: petroleum ether $/$ ethyl acetate $=$ 2/1). Mp 44-46 ${ }^{\circ} \mathrm{C} .{ }^{1} \mathrm{H}$ NMR $\left(400 \mathrm{MHz}, \mathrm{CDCl}_{3}\right) \delta 7.62-$ $7.56(\mathrm{~m}, 1 \mathrm{H}), 7.47(\mathrm{td}, J=7.5,1.2 \mathrm{~Hz}, 1 \mathrm{H}), 7.39(\mathrm{dd}, J=7.7$, $1.4 \mathrm{~Hz}, 1 \mathrm{H}), 7.29(\mathrm{td}, J=7.6,1.4 \mathrm{~Hz}, 1 \mathrm{H}), 7.25-7.16(\mathrm{~m}$, $3 \mathrm{H}), 7.15-7.09(\mathrm{~m}, 4 \mathrm{H}), 7.00-6.92(\mathrm{~m}, 2 \mathrm{H}), 5.22$ (d, $J=16.9$ $\mathrm{Hz}, 1 \mathrm{H}), 4.86(\mathrm{~d}, J=16.9 \mathrm{~Hz}, 1 \mathrm{H}), 4.71(\mathrm{dd}, J=10.4,0.9 \mathrm{~Hz}$, $1 \mathrm{H}), 4.57(\mathrm{~d}, J=10.3 \mathrm{~Hz}, 1 \mathrm{H}), 3.56-3.32(\mathrm{~m}, 2 \mathrm{H}), 3.18-3.06$ $(\mathrm{m}, 0.41 \mathrm{H}), 2.36(\mathrm{ddd}, J=15.4,13.1,2.5 \mathrm{~Hz}, 0.40 \mathrm{H})], 2.09-$ $1.92(\mathrm{~m}, 1 \mathrm{H}), 1.77-1.56(\mathrm{~m}, 1 \mathrm{H}) \cdot \mathrm{ppm} .{ }^{13} \mathrm{C} \mathrm{NMR}(100 \mathrm{MHz}$, $\left.\mathrm{CDCl}_{3}\right) \delta 171.7,140.7,138.6,136.7,134.7,134.6,130.9$, $129.5,129.0,128.5,128.2,127.1,127.0,126.3,126.2,124.8$, 122.0, 119.4, 118.1, 114.4, 110.2, 75.5, 49.8, 49.7, 47.5, 27.1, $27.1(\mathrm{t}, J=10.1 \mathrm{~Hz}), 24.2(\mathrm{q}, J=19.2 \mathrm{~Hz}) \mathrm{ppm}$. IR (film) $\nu_{\max }$ 2918, 1639, 1460,1422,1390, 1350, 1306, 1265, 1098 1056, $1028,1013,761,730,695 \mathrm{~cm}^{-1}$. HRMS (ESI) $\mathrm{m} / z$ calcd for $\mathrm{C}_{27} \mathrm{H}_{22} \mathrm{D}_{4} \mathrm{~N}_{2} \mathrm{NaO}_{2}$ 437.2138 $[\mathrm{M}+\mathrm{Na}]^{+}$, found 437.2138.

\section{ASSOCIATED CONTENT}

\section{Supporting Information}

The Supporting Information is available free of charge at https://pubs.acs.org/doi/10.1021/acsomega.1c04261.

Copies of ${ }^{1} \mathrm{H}$ and ${ }^{13} \mathrm{C}$ NMR spectra of all new compounds (PDF)

\section{Accession Codes}

CCDC 2087324 contains the supplementary crystallographic data for this paper. These data can be obtained free of charge via www.ccdc.cam.ac.uk/data_request/cif, or by emailing data_request@ccdc.cam.ac.uk, or by contacting The Cambridge Crystallographic Data Centre, 12 Union Road, Cambridge CB2 1EZ, U.K.; fax: +44 1223336033.

\section{AUTHOR INFORMATION}

\section{Corresponding Authors}

Huili Liu - State Key Laboratory of Magnetic Resonance and Atomic and Molecular Physics, National Center for Magnetic Resonance in Wuhan, Innovation Academy for Precision Measurement Science and Technology, Chinese Academy of Sciences, Wuhan 430071, China; Email: liuhuili@ wipm.ac.cn

Shaozhong Wang - State Key Laboratory of Coordination Chemistry, Jiangsu Key Laboratory of Advanced Organic Materials, School of Chemistry and Chemical Engineering, Nanjing University, Nanjing 210023, China; (1) orcid.org/ 0000-0002-7766-4433; Email: wangsz@nju.edu.cn

\section{Author}

Jianhui Qiao - State Key Laboratory of Coordination Chemistry, Jiangsu Key Laboratory of Advanced Organic Materials, School of Chemistry and Chemical Engineering, Nanjing University, Nanjing 210023, China

Complete contact information is available at: https://pubs.acs.org/10.1021/acsomega.1c04261

\section{Notes}

The authors declare no competing financial interest.

\section{ACKNOWLEDGMENTS}

The work was financially supported by the National Natural Science Foundation of China $(21871132,21572098)$ and the National Key Research and Development Program of China (2018YFC0310900).

\section{REFERENCES}

(1) Riemer, B.; Hofer, O.; Greger, H. Tryptamine Derived Amides from Clausena indica. Phytochemistry 1997, 45, 337-341.

(2) Steyn, P. S. The Structures of Five Diketopiperazines from Aspergillus ustus. Tetrahedron 1973, 29, 107-120.

(3) Hayashi, H.; Furutsuka, K.; Shiono, Y. Okaramines H and I, New Okaramine Congeners, from Aspergillus aculeatus. J. Nat. Prod. 1999, $62,315-317$.

(4) (a) Kam, T. S.; Yoganathan, K.; Chuah, C. H. Lundurines A, B and $\mathrm{C}$, new indole alkaloids with a novel carbon skeleton containing a cyclopropyl moiety. Tetrahedron Lett. 1995, 36, 759-762. (b) Kam, T. S.; Lim, K. H.; Yoganathan, K.; Hayashi, M.; Komiyama, K. Lundurines A-D, cytotoxic indole alkaloids incorporating a cyclopropyl moiety from Kopsia tenuis and revision of the structures of tenuisines A-C. Tetrahedron 2004, 60, 10739-10745.

(5) Neuss, N.; Neuss, M. N. In The Alkaloids; Brossi, A.; Suffness, M., Eds.; Academic: San Diego, 1990; Vol. 37, p 229. 
(6) Illuminati, G.; Mandolini, L. Ring Closure Reactions of Bifunctional Chain Molecules. Acc. Chem. Res. 1981, 14, 95-102.

(7) For selected cycloaddition reactions, see: (a) Wang, Y.-N.; Yang, L.-C.; Rong, Z.-Q.; Liu, T.-L.; Liu, R.; Zhao, Y. Pd-Catalyzed Enantioselective [6+4] Cycloaddition of Vinyl Oxetanes with Azadienes to Access Ten-Membered Heterocycles. Angew. Chem., Int. Ed. 2018, 57, 1596-1600. (b) Wu, A.; Feng, Q.; Sung, H. H. Y.; Williams, I. D.; Sun, J. Synthesis of Eight-Membered Lactams through Formal [6+2] Cyclization of Siloxy Alkynes and Vinylazetidines. Angew. Chem., Int. Ed. 2019, 58, 6776-6780. (c) Boyd, O.; Wang, G.W.; Sokolova, O. O.; Calow, A. D. J.; Bertrand, S. M.; Bower, J. F. Modular Access to Eight-Membered N-Heterocycles by Directed Carbonylative C-C Bond Activation of Aminocyclopropanes. Angew. Chem., Int. Ed. 2019, 58, 18844-18848.

(8) For selected cycloisomerization reactions, see: (a) Baran, P. S.; Corey, E. J. A Short Synthetic Route to (+)-Austamide, (+)-Deoxyisoaustamide, and $(+)$-Hydratoaustamide from a Common Precursor by a Novel Palladium-Mediated Indole $\rightarrow$ Dihydroindoloazocine Cyclization. J. Am. Chem. Soc. 2002, 124, 7904-7905. (b) Baran, P. S.; Guerrero, C. A.; Corey, E. J. Short, Enantioselective Total Synthesis of Okaramine N. J. Am. Chem. Soc. 2003, 125, 5628-5629. (c) Kirillova, M. S.; Muratore, M. E.; Dorel, R.; Echavarren, A. M. Concise Total Synthesis of Lundurines A-C Enabled by Gold Catalysis and a Homodienyl Retro-Ene/Ene Isomerization. J. Am. Chem. Soc. 2016, 138, 3671-3674. (d) Miloserdov, F. M.; Kirillova, M. S.; Muratore, M. E.; Echavarren, A. M. Unified Total Synthesis of Pyrroloazocine Indole Alkaloids Sheds Light on Their Biosynthetic Relationship. J. Am. Chem. Soc. 2018, 140, 5393-5400. (e) Zhou, B.; Li, L.; Zhu, X.-Q.; Yan, J.-Z.; Guo, Y.-L.; Ye, L.-W. Yttrium-Catalyzed Intramolecular Hydroalkoxylation/Claisen Rearrangement Sequence: Efficient Synthesis of Medium-Sized Lactams. Angew. Chem., Int. Ed. 2017, 56, 4015-4019. (f) Zhou, B.; Zhang, Y.-Q.; Zhang, K.; Yang, M.-Y.; Chen, Y.-B.; Li, Y.; Peng, Q.; Zhu, S.-F.; Zhou, Q.-L.; Ye, L.-W. Stereoselective synthesis of medium lactams enabled by metal-free hydroalkoxylation/stereospecific [1,3]-rearrangement. Nat. Commun. 2019, 10, No. 3234.

(9) For selected ring-expansion reactions, see (a) Ishikawa, H.; Colby, D. A.; Boger, D. L. Direct Coupling of Catharanthine and Vindoline to Provide Vinblastine: Total Synthesis of (+)- and ent(-)-Vinblastine. J. Am. Chem. Soc. 2008, 130, 420-421. (b) Gotoh, H.; Sears, J. E.; Eschenmoser, A.; Boger, D. L. New Insights into the Mechanism and an Expanded Scope of the Fe(III)-Mediated Vinblastine Coupling Reaction. J. Am. Chem. Soc. 2012, 134, 13240-13243. (c) Ishikawa, H.; Colby, D. A.; Seto, S.; Va, P.; Tam, A.; Kakei, H.; Rayl, T. J.; Hwang, I.; Boger, D. L. Total Synthesis of Vinblastine, Vincristine, Related Natural Products, and Key Structural Analogues. J. Am. Chem. Soc. 2009, 131, 4904-4916. (d) Beatty, J. W.; Stephenson, C. R. J. Synthesis of (-)-Pseudotabersonine, (-)-Pseudovincadifformine, and (+)-Coronaridine Enabled by Photoredox Catalysis in Flow. J. Am. Chem. Soc. 2014, 136, 10270-10273. (e) Zhang, L.; Wang, Y.; Yao, Z. J.; Wang, S.; Yu, Z. X. Kinetic or Dynamic Control on a Bifurcating Potential Energy Surface? An Experimental and DFT Study of Gold-Catalyzed Ring Expansion and Spirocyclization of 2-Propargyl- $\beta$-tetrahydrocarbolines. J. Am. Chem. Soc. 2015, 137, 13290-13300. (f) Huang, L.; Dai, L.-X.; You, S.-L. Enantioselective Synthesis of Indole-Annulated MediumSized Rings. J. Am. Chem. Soc. 2016, 138, 5793-5796. (g) Hall, J. E.; Matlock, J. V.; Ward, J. W.; Gray, K. V.; Clayden, J. Medium-Ring Nitrogen Heterocycles through Migratory Ring Expansion of Metalated Ureas. Angew. Chem., Int. Ed. 2016, 55, 11153-11157. (h) Costil, R.; Lefebvre, Q.; Clayden, J. Medium-Sized-Ring Analogues of Dibenzodiazepines by a Conformationally Induced Smiles Ring Expansion. Angew. Chem., Int. Ed. 2017, 56, 1460214606. (i) Wang, N.; Gu, Q.-S.; Li, Z.-L.; Li, Z.; Guo, Y.-L.; Guo, Z.; Liu, X.-Y. Direct Photocatalytic Synthesis of Medium-Sized Lactams by C-C Bond Cleavage. Angew. Chem., Int. Ed. 2018, 57, 1422514229. (j) Lawer, A.; Rossi-Ashton, J. A.; Stephens, T. C.; Challis, B. J.; Epton, R. G.; Lynam, J. M.; Unsworth, W. P. Internal Nucleophilic Catalyst Mediated Cyclisation/Ring Expansion Cascades for the
Synthesis of Medium-Sized Lactones and Lactams. Angew. Chem., Int. Ed. 2019, 58, 13942-13947.

(10) (a) Qiao, J.; Zhao, W.; Liang, Y.; Yao, Z.-J.; Wang, S. Diastereose-lective Access to Tetracyclic Eight-Membered Lactams through a Dearomative Heck Reaction and an Alkylative RingOpening Driven by the Photoexcited Spiroindolines. Chem. - Eur. J. 2021, 27, 6308-6314. (b) Li, H.-H.; Ye, S.-H.; Chen, Y.-B.; Luo, W.F.; Qian, P.-C.; Ye, L.-W. Efficient and Divergent Synthesis of Medium-Sized Lactams through Zinc-Catalyzed Oxidative Cyclization of Indoly Ynamides. Chin. J. Chem. 2020, 38, 263-268. (c) Zhu, B.H.; Zheng, Y.-X.; Kang, W.; Deng, C.; Zhou, J.-M.; Ye, L.-W. Catalytic hydrative cyclization of aldehyde-ynamide with water for synthesis of medium-sized lactams. China: Chem. 2021, DOI: 10.1007/s11426021-1069-7.

(11) For selected reviews on $\mathrm{C}-\mathrm{C}$ bond fragmentation, see: (a) Morcillo, S. P. Radical-Promoted C-C Bond Cleavage: A Deconstructive Approach for Selective Functionalization. Angew. Chem., Int. Ed. 2019, 58, 14044-14054. (b) Drahl, M. A.; Manpadi, M.; Williams, L. J. C-C Fragmentation: Origins and Recent Applications. Angew. Chem., Int. Ed. 2013, 52, 11222-11251. (c) Prantz, K.; Mulzer, J. Synthetic Applications of the Carbonyl Generating Grob Fragmentation. Chem. Rev. 2010, 110, 3741-3766. (d) Grob, C. A. Mechanisms and Stereochemistry of Heterolytic Fragmentation. Angew. Chem., Int. Ed. 1969, 8, 535-546.

(12) Atzrodt, J.; Derdau, V.; Kerr, W. J.; Reid, M. Deuterium and Tritium-Labelled Compounds: Applications in the Life Sciences. Angew. Chem., Int. Ed. 2018, 57, 1758-1784. 